\title{
Synthesis and X-ray crystal structures of $\beta$-ketoiminate complexes
}

\author{
Phillip Steiniger, Dennis Dittrich, Christoph Scheiper, Christoph John, Christoph Wölper, and Stephan \\ Schulz*
}

Dedicated to Prof. W. Uhl on the occasion of his 65th birthday

\begin{abstract}
Heteroleptic zinc complexes containing side-arm donorfunctionalized tridentate $\beta$-ketoiminate ligands were obtained from metalation reactions of $\beta$-ketoimines $\mathrm{L} 1 / 2 / 3^{\mathrm{D} 01 / 2 / 3 / 4} \mathrm{H} \quad(\mathrm{L} 1=$ $\left\{\left(\mathrm{OC}(\mathrm{Me}) \mathrm{C}(\mathrm{H}) \mathrm{C}(\mathrm{Me}) \mathrm{NCH}_{2} \mathrm{CH}_{2} \mathrm{Do}\right)\right\}, \quad \mathrm{L} 2=\{(\mathrm{OC}(\mathrm{Me}) \mathrm{C}(\mathrm{H}) \mathrm{C}(\mathrm{Me})-$ $\left.\left.\mathrm{NCH}_{2} \mathrm{CH}_{2} \mathrm{CH}_{2} \mathrm{Do}\right)\right\}, \mathrm{L} 3=\left\{\left(\mathrm{OC}(\mathrm{OMe}) \mathrm{C}(\mathrm{H}) \mathrm{C}(\mathrm{Me}) \mathrm{NCH}_{2} \mathrm{CH}_{2} \mathrm{Do}\right)\right\} ; \mathrm{Do} 1=$ $\mathrm{NMe}_{2}$, Do2 $=\mathrm{NEt}_{2} ; \mathrm{Do} 3=\mathrm{Pyr}$, Do4 $=\mathrm{OMe}$ ) with equimolar amounts of $\mathrm{Zn}\left[\mathrm{N}\left(\mathrm{SiMe}_{3}\right)_{2}\right]_{2}\left(\mathrm{~L}^{\mathrm{Do}}{ }^{\mathrm{Dn}} \mathrm{ZnN}\left(\mathrm{SiMe}_{3}\right)_{2} \quad \mathbf{1}, \mathrm{L}^{\mathrm{Do}}{ }^{\mathrm{Zn}} \mathrm{ZnN}\left(\mathrm{SiMe}_{3}\right)_{2} 2\right)$ and

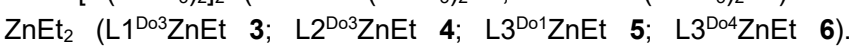
Analogous reactions of $\mathrm{AlMe}_{3}$ and $\mathrm{Cp}^{*} \mathrm{MMe}_{3}$ with $\mathrm{L} 1^{\mathrm{Do} 1 / 2} \mathrm{H}$ yielded homoleptic aluminum ( $\mathrm{L}^{\mathrm{Do} 01} \mathrm{AlMe}_{2} 7$; L1 ${ }^{\mathrm{Do} 2} \mathrm{AIMe}_{2}$ 8; L2 ${ }^{\mathrm{Do1}} \mathrm{AIMe}_{2}$ 9), titanium $\left(\mathrm{L}^{\mathrm{D} 02} \mathrm{Ti}\left(\mathrm{Me}_{2}\right) \mathrm{Cp}^{*} \quad 10\right)$ and zirconium complexes $\left(\mathrm{L} 1^{\mathrm{Do}} \mathrm{Zr}\left(\mathrm{Me}_{2}\right) \mathrm{Cp}^{*}\right.$ 11). In addition, 7 reacts with an ethereal $\mathrm{HCl}$ solution with methane elimination and formation of $\mathrm{L}^{\mathrm{D} 01} \mathrm{Al}(\mathrm{Cl}) \mathrm{Me}$ (12) and $\mathrm{L}_{1}{ }^{\mathrm{Do} 1} \mathrm{AlCl}_{2}$ (13), while the reaction with one and two equivalents of 2,6-dimethylphenol yielded $\mathrm{L}^{\mathrm{Do}}{ }^{\mathrm{Al}} \mathrm{Al}(\mathrm{Me}) \mathrm{O}-2,6$ $\mathrm{Me}_{2} \mathrm{C}_{6} \mathrm{H}_{3}$ (14) and $\mathrm{L} 1^{\mathrm{Do} 1} \mathrm{Al}\left(\mathrm{O}-2,6-\mathrm{Me}_{2} \mathrm{C}_{6} \mathrm{H}_{3}\right)_{2}$ (15). The complexes were characterized by elemental analysis, ${ }^{1} \mathrm{H},{ }^{13} \mathrm{C}$ NMR and IR spectroscopy and single crystal $X$-ray analysis $(2,4,7,10,12-14)$.
\end{abstract}

\section{Introduction}

Chelating N,O ligands are well established in coordination chemistry. Aside from classical bidentate $\mathrm{N}, \mathrm{O}$ ligands such as $\mathrm{N}, \mathrm{O}$-chelating $\beta$-ketoimines (I) and aminophenoles (II), donorfunctionalized tridentate-type O,N,N (III), and O,N,O (IV) ligands as shown in scheme 1 have also been investigated in detail. ${ }^{[1]}$ In the last two decades, these type of ligands have also been extensively applied for the synthesis of a large variety of transition metal and main group metal catalysts for the ring opening polymerization (ROP) of cyclic ethers including lactide and $\varepsilon$-caprolactone, respectively. ${ }^{[2]}$ In particular catalysts based on rather cheap and non-toxic metals such as $\mathrm{Mg}, \mathrm{Ti}, \mathrm{Zn}$ and $\mathrm{Al}$ as well as lanthanide metals have been widely applied. ${ }^{[3]}$

$\beta$-ketoimines (type I) are very promising ligands in main group metal and transition metal chemistry since their steric and electronic properties can be effectively controlled to a very large extent. The steric demand of $\beta$-ketoimines can easily by

[a] P. Steiniger, Dr. D. Dittrich, Dr. C. Scheiper, C. John, Dr. C. Wölper, Prof. Dr. S. Schulz

Faculty of Chemistry and Center for Nanointegration DuisburgEssen (CENIDE)

University of Duisburg-Essen

45117 Essen, Germany

E-mail: stephan.schulz@uni-due.de

Supporting information for this article is given via a link at the end of the document. modified by introducing substituents $\mathrm{R}$ and $\mathrm{R}$ ' with different sizes in the backbone as well as on the imine center by using different primary amines, ranging from those containing small substituents to those containing demanding organic groups. Analogous findings were reported for $\beta$-diketimines and other $\mathrm{N}, \mathrm{N}^{\prime}$-chelating substituents such as dipyrromethene ligands. The steric demand of the specific ligand not only results in a kinetic stabilization of the complexes, ${ }^{[4]}$ which sometimes become even air-stable, but also dramatically influences the activity for the stereo-regular ROP of lactide as was demonstrated by Coates et al. for specific $\beta$-diketiminate zinc complexes. ${ }^{[5]}$ In addition, the electronic properties of $\beta$-ketoimines can be controlled by using electron donating or electron withdrawing substituents $R$ and $\mathrm{R}^{\prime}$, as was also demonstrated for $\beta$-diketimines. ${ }^{[6]}$ Moreover, the introduction of a side-arm donor function (Do) in $\beta$-ketoimines of type $\mathbf{V}$ can further influence the structure of the resulting complexes in solution and in the solid state such as their tendency toward dimerization as well as their tendency toward ligand disproportionation reaction as was often observed for heteroleptic zinc and aluminum complexes. ${ }^{[7]}$

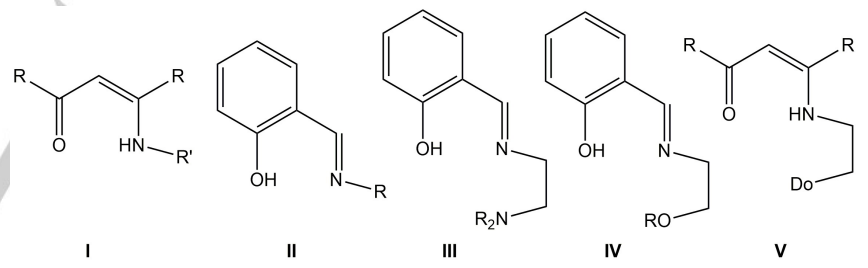

Scheme 1. Typical bidentate $N, O$ and tridentate $O, N, N$ and $O, N, O$ ligands.

We recently reported on the solid state structures of monomeric $\beta$-ketoiminate complexes $\mathrm{L} 2{ }^{\mathrm{Do1}} \mathrm{ZnR}(\mathrm{R}=\mathrm{Me}, \mathrm{Et}){ }^{[8]}$ In contrast, complex $\mathrm{L} 1^{\mathrm{Do} 2} \mathrm{ZnEt}$ was reported to form a tetrameric structure in the solid state. ${ }^{[9]}$ Unfortunately, any attempts to structurally characterize the complexes $\mathrm{L} 1 \mathrm{Do}^{1 / 2} \mathrm{ZnMe}$ and $\mathrm{L} 1^{\mathrm{D} 01} \mathrm{ZnEt}$ as well as $L 2{ }^{\mathrm{Do} 2} \mathrm{ZnR}\left(\mathrm{R}=\mathrm{Me}\right.$, Et) failed. ${ }^{[8]}$ To further investigate the influence of $\beta$-ketoimininate ligand, in particular of the side-arm donor group, and of the $\mathrm{Zn}$-bonded group on the structure of the resulting complexes in the solid state in more detail, we now synthesized different donor-functionalized $\beta$-ketoimines $\mathrm{LH}$ and reacted them with either $\mathrm{Zn}\left[\mathrm{N}\left(\mathrm{SiMe}_{3}\right)_{2}\right]_{2}$ or $\mathrm{ZnEt}_{2}$. In addition, we extended our studies on reactions of such $\beta$-ketoimines $\mathrm{LH}$ with other main group metal and transition metal alkyl of group $4(\mathrm{Ti}$, $\mathrm{Zr})$ and $13(\mathrm{Al})$, respectively, since these metals are also of potential interest in ROP of cyclic ethers. 


\section{Results and Discussion}

Six donor-functionalized $\beta$-ketoimines LH (Scheme 1) were synthesized by condensation reaction between the corresponding diketone (acetylacetone, methyl acetoacetate) and the primary amine ( $\mathrm{N}, \mathrm{N}$-dimethylethylenediamine, N,Ndiethylethylenediamine, 1-(3-aminopropyl)pyrrolidine, methoxyethylamine) according to literature known methods. ${ }^{[10]}$<smiles>CC1=C[CH]N(CCO)C(C)=C1</smiles><smiles>CC1=C[CH]N(CCCO)C(C)=C1</smiles><smiles>COC1=CC(C)=C[CH]N1CCO</smiles>

$$
\begin{aligned}
& \mathrm{Do}=\mathrm{NMe}_{2}, \mathbf{L 1}^{\mathrm{Do1}} \\
& \text { Do }=\mathrm{NEt}_{2}, \mathbf{L 1}^{\mathbf{D} \mathbf{2} 2} \\
& \text { Do }=\mathrm{Pyr}_{\mathbf{L}} \mathbf{L 1}^{\mathbf{D o 3}}
\end{aligned}
$$

geometries as was previously observed for zinc complexes containing donor-functionalized $\beta$-ketoiminate ligands. ${ }^{[8]}$

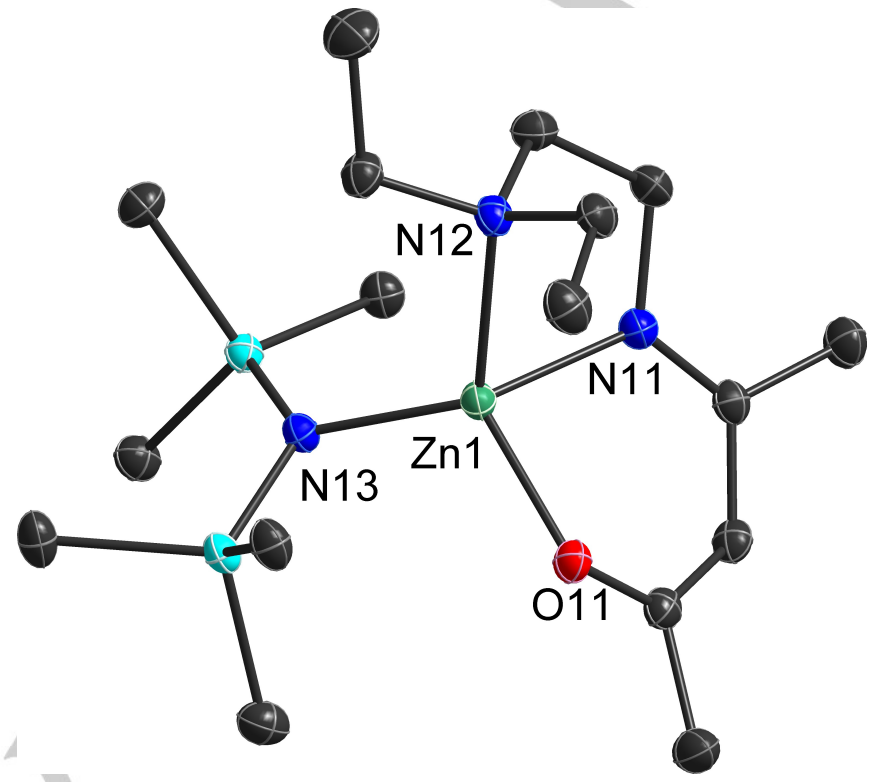

Figure 1. Crystal structure of $\mathrm{L}^{\mathrm{Do}}{ }^{\mathrm{D}} \mathrm{ZnN}\left(\mathrm{SiMe}_{3}\right)_{2}$ (2). All hydrogen atoms have been omitted for clarity. Ellipsoids are drawn to a $50 \%$ probability level. Only

one of the two independent molecules is displayed.

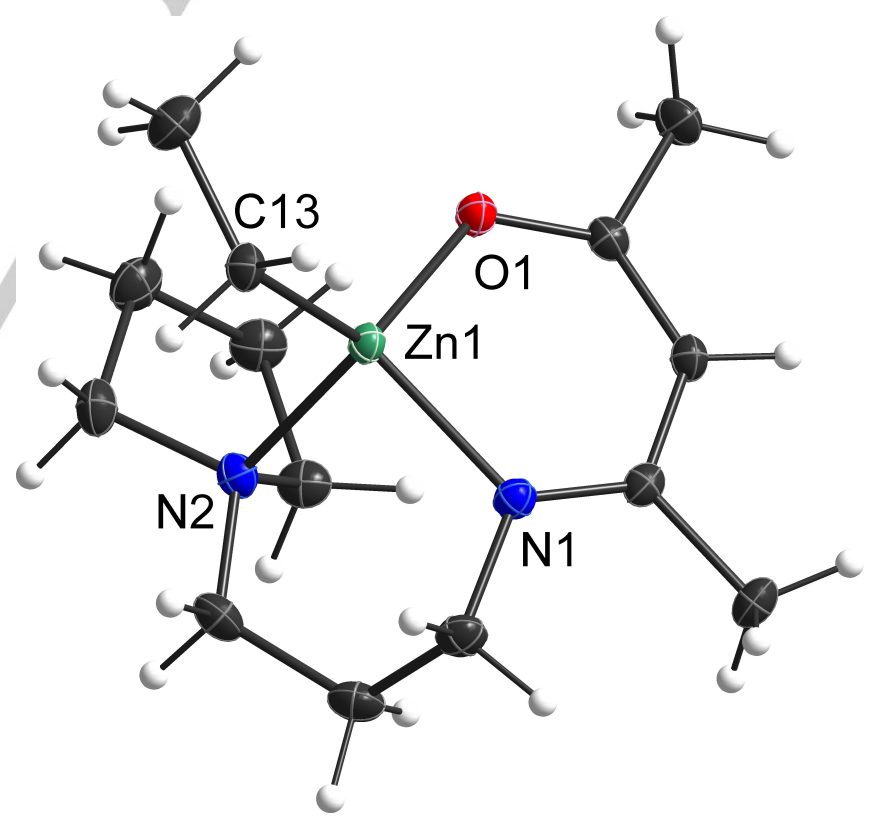

Figure 2. Crystal structure of $\mathrm{L}^{\mathrm{D}}{ }^{\circ} \mathrm{ZnEt}$ (4). Ellipsoids are drawn to a $50 \%$ probability level and hydrogen atoms as spheres of arbitrary radii. Unfortunately, single crystals suitable for X-ray analyses were only obtained for $\mathbf{2}$ and $\mathbf{4}$ upon storage of solutions of $\mathbf{2}$ and $\mathbf{4}$ in $n$-hexane at $-30^{\circ} \mathrm{C} \cdot{ }^{[11]} \mathbf{2}$ and $\mathbf{4}$ form monomeric structures in the solid state (Figures 1 and 2) and their central structural parameters are almost identical (Table 1). The zinc atoms in 2 and 4 adopt slightly distorted tetrahedral coordination

yellow oils in almost quantitative yields after workup. Their ${ }^{1} \mathrm{H}$ and ${ }^{13} \mathrm{C}$ NMR spectra show the expected resonances of the acetylacetone backbone, the $\mathrm{C}_{2} \mathrm{H}_{4}$ or $\mathrm{C}_{3} \mathrm{H}_{6}$ spacer and the specific donor groups (Do $=\mathrm{NMe}_{2}, \mathrm{NEt}_{2}, \mathrm{Pyr}, \mathrm{OMe}$ ). $\mathrm{Zn}\left[\mathrm{N}\left(\mathrm{SiMe}_{3}\right)_{2}\right]_{2}$ and $\mathrm{ZnEt}_{2}$, respectively. The corresponding obtained in high yields (>80 \%) after workup.<smiles>CC1=CC(C)=N[C@]2(NN)OCCN12</smiles>

$\mathrm{Do}=\mathrm{NMe}_{2}(\mathbf{1})$ $\mathrm{Do}=\mathrm{NEt}_{2}(2)$<smiles>CCC12C=C(C)C=C(C)N1CCO2</smiles>

Do $=\operatorname{Pyr}(3)$
Do $=\operatorname{Pyr}(\mathbf{4})$
$\mathrm{Do}=\mathrm{NMe}_{2}(\mathbf{5})$ $\mathrm{Do}=\mathrm{OMe}(\mathbf{6})$
Scheme 3. Drawing of the heteroleptic zinc complexes 1 - 6.

${ }^{1} \mathrm{H}$ and ${ }^{13} \mathrm{C}$ NMR spectra of the heteroleptic complexes $1-6$ show the resonances of the $\beta$-ketoiminate ligands and the $\mathrm{ZnR}$ group $\left(\mathrm{R}=\mathrm{N}\left(\mathrm{SiMe}_{3}\right)_{2} \mathbf{1}\right.$, 2; Et $\mathbf{3}$ - 6) in the expected 1:1 molar ratio. In contrast, the homoleptic complex $\left(\mathrm{L}^{\mathrm{Do} 04}\right)_{2} \mathrm{Zn} 7$ only shows the reson, the $\beta$ keto
The Zn-O (2 1.9815(12), 1.9769(12) A; 4 1.9767(8) A) and Zn-N bond lengths (2 1.9943(14), 1.9949(15) $\AA ; 4$ 2.0050(10) $\AA$ ) as well as the $\mathrm{N}-\mathrm{C}, \mathrm{C}-\mathrm{C}$ and $\mathrm{O}-\mathrm{C}$ bond lengths and $\mathrm{N}-\mathrm{C}-\mathrm{C}, \mathrm{C}-\mathrm{C}-\mathrm{C}$ and $\mathrm{C}-\mathrm{C}-\mathrm{O}$ bond angles within the six-membered $\mathrm{ZnNOC}_{3}$ rings 
are almost identical (Table 1). Similar bond lengths and angles were observed in zinc complexes containing tridentate $\beta$ ketoiminate, amino- and iminophenolate ligands. ${ }^{[12]}$ In contrast, the endocyclic 01-Zn1-N1 bond angles (2 92.03(5), 92.09(6), 4 $\left.94.89(4)^{\circ}\right)$ significantly deviate from each other. The $\mathrm{Zn}-\mathrm{N}$ bond lengths toward the coordinating amine donor group (2 2.1849(14), 2.1648(14) $\AA ; 4$ 2.2031(10) $\AA$ ) are also comparable. In contrast, the side-arm donor groups of the homoleptic complexes $\left(L 1^{D 01}\right)_{2} Z n$ and $\left(L 1^{D 04}\right)_{2} Z n$ do not coordinate to the zinc atom, ${ }^{[13]}$ since the tetrahedral coordination geometry of the central zinc atom is already achieved by coordination of two $\mathrm{N}, \mathrm{O}$-chelating $\beta$-ketoiminate ligands. In contrast, the zinc atom in the related homoleptic zinc complex $\left(\mathrm{L}^{\mathrm{Do1}}\right)_{2} \mathrm{Zn} \quad\left(\mathrm{L}^{\mathrm{Do1}}=\right.$ $\left.\left\{\left(\mathrm{OC}(\mathrm{Ph}) \mathrm{C}(\mathrm{H}) \mathrm{C}(\mathrm{Me}) \mathrm{NCH}_{2} \mathrm{CH}_{2} \mathrm{Do}\right)\right\}\right)$, in which one Me group of the $\beta$-ketoiminate ligand is replaced by a $\mathrm{Ph}$ group, is additionally coordinated by one side-arm donor group, resulting in a distorted trigonal-bipyramidal coordination geometry. ${ }^{[14]}$

Table 1. Selected bond lengths $(\AA)$ and bond angles $\left({ }^{\circ}\right)$ of $L 1^{\mathrm{Do}} \mathrm{ZnN}\left(\mathrm{SiMe}_{3}\right)_{2}$ (2) and L2 ${ }^{\mathrm{Do} 3} \mathrm{ZnEt}(\mathbf{4})$

\begin{tabular}{|c|c|c|}
\hline & $2^{[a]}$ & 4 \\
\hline $\mathrm{Zn}-\mathrm{N}$ & $1.9943(14), 1.9949(15)$ & $2.0050(10)$ \\
\hline $\mathrm{Zn}-\mathrm{O}$ & $1.9815(12), 1.9769(12)$ & $1.9767(8)$ \\
\hline $\mathrm{Zn}-\mathrm{N}_{(\mathrm{amin})}$ & $2.1849(14), 2.1648(14)$ & $2.2031(10)$ \\
\hline $\mathrm{Zn}-\mathrm{N}_{(\mathrm{tms})}$ & $1.9280(14), 1.9314(14)$ & - \\
\hline $\mathrm{Zn}-\mathrm{C}$ & - & $1.9904(11)$ \\
\hline $\mathrm{N} 1-\mathrm{C} 1$ & $1.304(2), 1.307(2)$ & $1.3082(15)$ \\
\hline O1-C3 & $1.292(2), 1.296(2)$ & $1.2895(14)$ \\
\hline C1-C2 & $1.427(2), 1.422(3)$ & $1.4273(16)$ \\
\hline C2-C3 & $1.381(2), 1.380(3)$ & $1.3845(16)$ \\
\hline $\mathrm{N}-\mathrm{Zn}-\mathrm{O}$ & $92.03(5), 92.09(6)$ & $94.89(4)$ \\
\hline $\mathrm{N}_{(\mathrm{amin})}-\mathrm{Zn}-\mathrm{O}$ & $122.24(5), 125.19(6)$ & $104.50(4)$ \\
\hline $\mathrm{N}-\mathrm{Zn}-\mathrm{N}_{(\mathrm{amin})}$ & $81.29(6), 81.52(6)$ & $88.41(4)$ \\
\hline Zn1-N1-C1 & $123.25(12), 122.61(13)$ & $124.13(8)$ \\
\hline Zn1-O1-C3 & $118.72(11), 116.72(12)$ & $121.90(8)$ \\
\hline $\mathrm{C} 1-\mathrm{C} 2-\mathrm{C} 3$ & $126.02(16), 126.27(17)$ & $128.04(10)$ \\
\hline
\end{tabular}

[a] two independent molecules

Since titanium and aluminum complexes are also of potential interest as catalyst for the ROP of cyclic ethers, we became further interested to extend our studies on the synthesis of heteroleptic $\beta$-ketoiminate complexes of the general type $\mathrm{LAIR}_{2}$ and $\mathrm{LMR}_{3}(\mathrm{R}=\mathrm{Ti}, \mathrm{Zr})$, respectively. The reaction of $\mathrm{AlMe}_{3}$ with $\mathrm{LH}$ at low temperature proceeded with smooth elimination of methane and subsequent formation of the heteroleptic aluminum complexes $\mathrm{L} 1^{\mathrm{Do}} \mathrm{AlMe}_{2}$ (7), which has been prepared previously, ${ }^{[15]} \mathrm{L}^{\mathrm{Do}}{ }^{\mathrm{Al}} \mathrm{AlMe}_{2}$ (8) and $\mathrm{L}^{\mathrm{D}}{ }^{\mathrm{o} 1} \mathrm{AlMe}_{2}$ (9), respectively. Comparable results were obtained in the reactions of $\mathrm{LH}$ with
$\mathrm{Cp}^{*} \mathrm{MMe}_{3}$, which also occurred with methane elimination and almost quantitative formation of the heteroleptic complexes $\mathrm{L} 1^{\mathrm{D} 01} \mathrm{Ti}\left(\mathrm{Cp}^{*}\right) \mathrm{Me}_{2}(\mathbf{1 0})$ and L1 ${ }^{\mathrm{Do} 1} \mathrm{Zr}\left(\mathrm{Cp}^{*}\right) \mathrm{Me}_{2}$ (11) (Scheme 3).

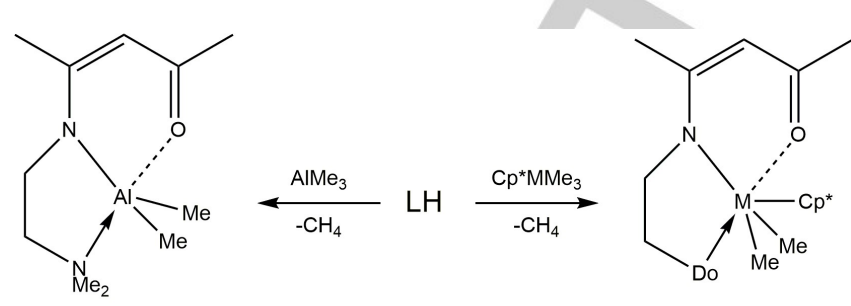

$\mathrm{L}^{\mathrm{Dol}} \mathrm{AlMe}_{2}(7)$

$\mathrm{L}_{1}{ }^{\mathrm{Do} 2} \mathrm{AlMe}_{2}(\mathbf{8})$

$\mathrm{L}_{2}{ }^{\mathrm{Dol}} \mathrm{AlMe}_{2}(9)$

$\mathrm{L}^{\mathrm{Dol}} \mathrm{M}\left(\mathrm{Cp}^{*}\right) \mathrm{Me}_{2}$ $\mathrm{M}=\operatorname{Ti}(\mathbf{1 0}), \operatorname{Zr}(\mathbf{1 1})$

Scheme 4. Synthesis of heteroleptic aluminum and titanium $\beta$-ketiminate complexes $(7-11)$.

${ }^{1} \mathrm{H}$ and ${ }^{13} \mathrm{C}$ NMR spectra of the heteroleptic complexes $7-11$ show the resonances of the $\beta$-ketiminate ligands and the $\mathrm{MMe}_{2}$ groups $(\mathrm{M}=\mathrm{Al}, \mathrm{Ti}, \mathrm{Zr})$. In addition, the ${ }^{1} \mathrm{H}$ and ${ }^{13} \mathrm{C}$ NMR spectra of 10 and 11 each show one additional resonance of the Me substituent of the $\mathrm{Cp}^{*}$ ligand as well as one resonance of the ring carbon atoms $\left({ }^{13} \mathrm{C}\right.$ NMR), indicating a $\eta^{5}$-coordination of the $\mathrm{Cp}^{*}$ ligand in solution.

Reactions of $\mathrm{L}^{\mathrm{Do}}{ }^{\mathrm{AlM}} \mathrm{Al}_{2}$ (7) with $\mathrm{HCl}$ in $\mathrm{Et}_{2} \mathrm{O}$ yielded $\mathrm{L} 1{ }^{\mathrm{Do}} \mathrm{Al}(\mathrm{Cl}) \mathrm{Me}$ (12), while $\mathrm{L}^{\mathrm{Do1}} \mathrm{AlCl}_{2}$ (13) was obtained by equimolar reaction of 12 with $\mathrm{GaCl}_{3} .12$ and 13 were isolated by fractional crystallization from solutions in $n$-hexane. 7 also reacted with one and two equivalents of $\mathrm{ROH}\left(\mathrm{R}=2,6-\mathrm{Me}_{2} \mathrm{C}_{6} \mathrm{H}_{3}\right)$ with elimination of ethane and formation of $\mathrm{L}^{\mathrm{Do1}} \mathrm{Al}(\mathrm{Me}) \mathrm{O}-2,6$ $\mathrm{Me}_{2} \mathrm{C}_{6} \mathrm{H}_{3}$ (14) and $\mathrm{L} 1{ }^{\mathrm{Do} 1} \mathrm{Al}\left(\mathrm{O}-2,6-\mathrm{Me}_{2} \mathrm{C}_{6} \mathrm{H}_{3}\right)_{2}$ (15) (Scheme 4). ${ }^{1} \mathrm{H}$ and ${ }^{13} \mathrm{C}$ NMR spectra of the heteroleptic complexes $12-15$ exhibit the resonances of the $\beta$-ketiminate ligand and the AIMe group $(12,14)$ as well as the 2,6 -dimethylphenoxide ligand in the expected 1:1 (14) and 1:2 (15) molar ratios.<smiles>CC(=O)C=C(C)N1CCN2C(C)=CC(C)=N[C@@]12Cl</smiles><smiles>CC1=CC(C)=N[N+]([O-])(O)O1</smiles><smiles></smiles>

$\mathrm{L}^{\mathrm{Dol}} \mathrm{Al}(\mathrm{Me}) \mathrm{OR}(\mathbf{1 4}) \quad \mathrm{L1}^{\mathrm{Dol}} \mathrm{Al}(\mathrm{OR})_{2}(15)$

Scheme 5. Drawing of the heteroleptic aluminum complexes (12 - 15)

Single crystals of the aluminum complexes 7 and $12-14$ (Figures 3-6) were obtained from solutions in $n$-hexane upon storage at $-30{ }^{\circ} \mathrm{C} .{ }^{[11]} 7$ and $12-14$ adopt monomeric structures in the solid state (Figures $4-7$ ). Their central structural parameters are summarized in table 2 . 


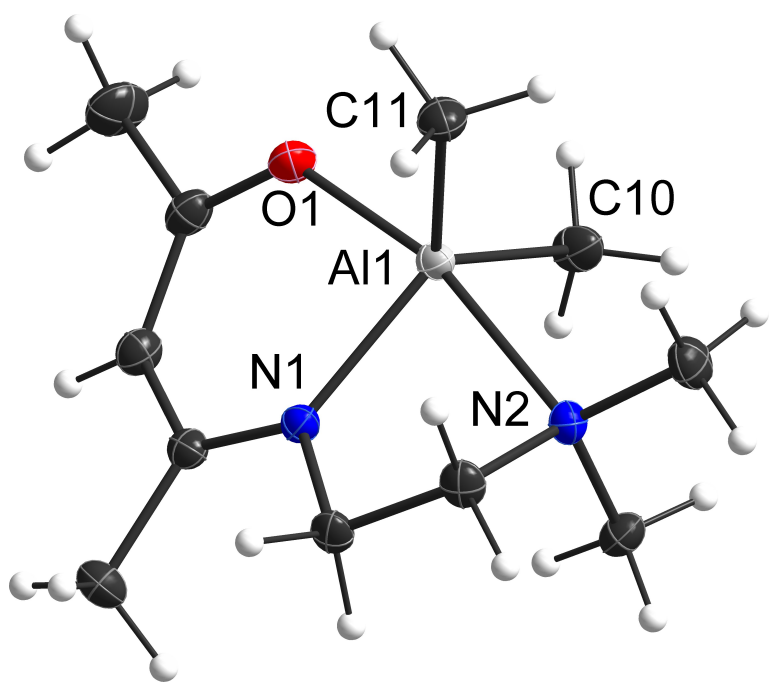

Figure 3. Crystal structure of $\mathrm{L} 1^{\mathrm{Do} 1} \mathrm{AIMe}_{2}$ (7). Ellipsoids are drawn to a $50 \%$ probability level and hydrogen atoms as spheres of arbitrary radii.

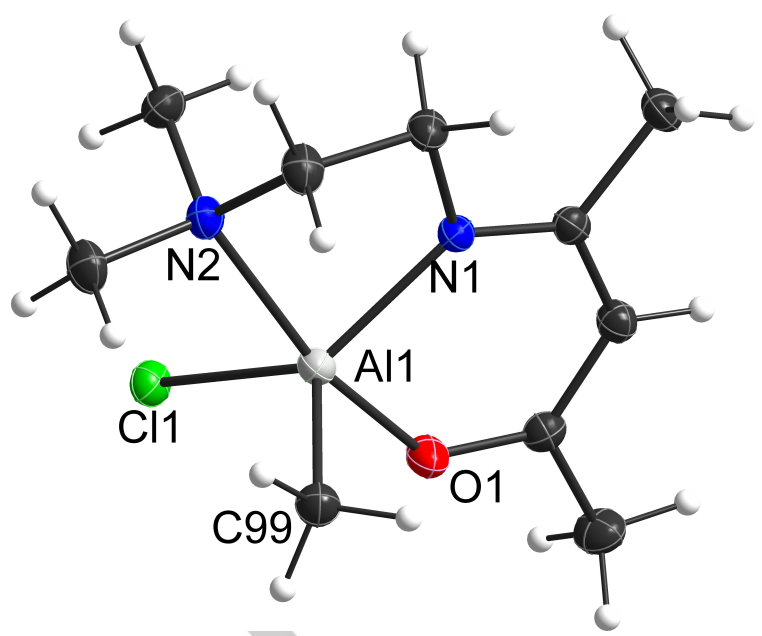

Figure 4. Crystal structure of $\mathrm{L} 1{ }^{\mathrm{Do} 1} \mathrm{Al}(\mathrm{Cl}) \mathrm{Me}$ (12). Solvent and minor part of the disorder have been omitted for clarity. Ellipsoids are drawn to a $50 \%$ probability level and hydrogen atoms as spheres of arbitrary radii.

Table 2. Selected bond lengths $(\AA)$ and bond angles $\left({ }^{\circ}\right)$ of $L 1^{D \circ 1} A I M e_{2}(7)$, $\mathrm{L} 1^{\mathrm{Do1}} \mathrm{Al}(\mathrm{Cl}) \mathrm{Me}$ (12), L1 ${ }^{\mathrm{Do1}} \mathrm{AlCl}_{2}$ (13) and L1 ${ }^{\mathrm{Do1}} \mathrm{Al}(\mathrm{Me}) \mathrm{O}-2,6-\mathrm{Me}_{2} \mathrm{C}_{6} \mathrm{H}_{3}$ (14).

\begin{tabular}{|c|c|c|c|c|}
\hline & 7 & 12 & 13 & 14 \\
\hline Al-N1 & $1.9857(7)$ & $1.9658(11)$ & $1.9414(6)$ & $1.9719(8)$ \\
\hline $\mathrm{Al}-\mathrm{O} 1$ & $1.8984(7)$ & $1.8541(11)$ & $1.8232(6)$ & $1.8656(7)$ \\
\hline Al-N2 & $2.2283(8)$ & $2.1468(12)$ & $2.0921(6)$ & $2.1441(9)$ \\
\hline $\mathrm{Al}-\mathrm{C}$ & $\begin{array}{l}1.9942(9) \\
1.9896(9)\end{array}$ & $2.036(7)^{[a]}$ & - & $36(11)$ \\
\hline $\mathrm{Al}-\mathrm{Cl}$ & - & $2.213(2)^{[a]}$ & $\begin{array}{l}2.2179(3) \\
2.1774(3)\end{array}$ & - \\
\hline $\mathrm{Al}-\mathrm{O}^{(\mathrm{ph})}$ & - & - & - & $1.7870(7)$ \\
\hline N1-C1 & $1.3233(10)$ & $1.3231(16)$ & $1.3234(9)$ & $1.3235(13)$ \\
\hline O1-C3 & $1.2827(11)$ & $1.2856(16)$ & $1.2939(9)$ & $1.2858(12)$ \\
\hline C1-C2 & $1.4156(11)$ & $1.4160(17)$ & $1.4173(10)$ & $1.4188(14)$ \\
\hline C2-C3 & $1.3838(12)$ & $1.3794(18)$ & $1.3760(10)$ & $1.3834(14)$ \\
\hline N1-Al-O1 & $88.65(3)$ & $89.92(5)$ & $91.15(3)$ & $89.23(3)$ \\
\hline $\mathrm{N}_{\text {amine}}-\mathrm{Al}-\mathrm{O} 1$ & $166.03(3)$ & $164.93(5)$ & $167.10(3)$ & $165.08(4)$ \\
\hline N1-Al-Namine & $77.72(3)$ & $79.92(4)$ & $80.88(3)$ & $79.16(4)$ \\
\hline Al-N1-C1 & $125.62(5)$ & $126.89(9)$ & $126.57(5)$ & $126.24(7)$ \\
\hline $\mathrm{Al}-\mathrm{O} 1-\mathrm{C} 3$ & $129.03(6)$ & $132.52(9)$ & $131.67(5)$ & $130.19(6)$ \\
\hline C1-C2-C3 & $123.35(8)$ & $123.56(12)$ & $123.84(6)$ & $123.74(9)$ \\
\hline
\end{tabular}

[a] Restraints applied

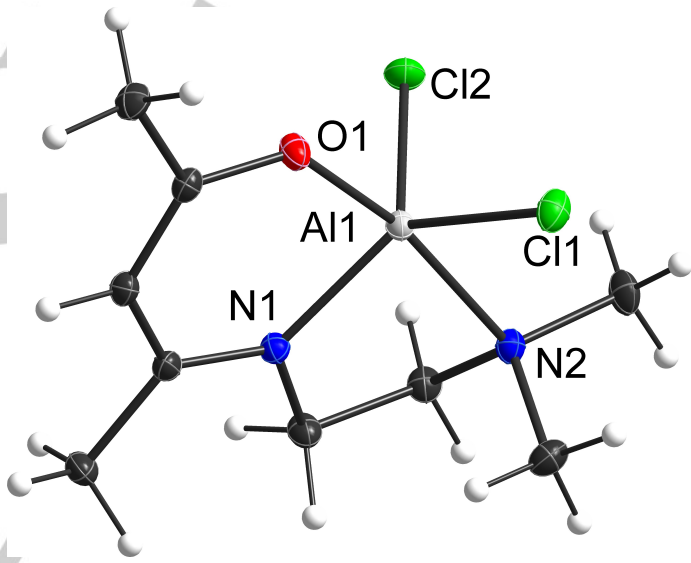

Figure 5. Crystal structure of $\mathrm{L}^{\mathrm{Do} 1} \mathrm{AlCl}_{2}$ (13). Ellipsoids are drawn to a $50 \%$ probability level and hydrogen atoms as spheres of arbitrary radii.

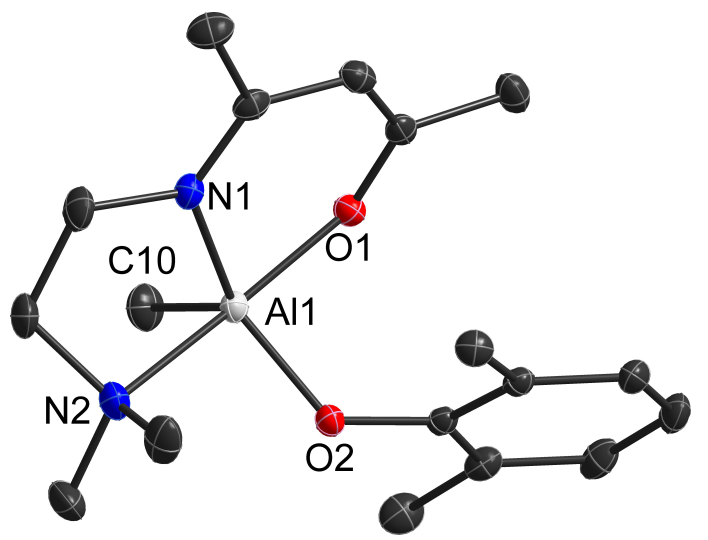

Figure 6. Crystal structure of $\mathrm{L}_{1}{ }^{\mathrm{Do}} \mathrm{Al}(\mathrm{Me}) \mathrm{O}-2,6-\mathrm{Me}_{2} \mathrm{C}_{6} \mathrm{H}_{3}$ (14). All hydrogen atoms have been omitted for clarity. Ellipsoids are drawn to a50 \% probability level. 
$\mathrm{L} 1^{\mathrm{Do}} \mathrm{AIMe}_{2} 7$ is a new polymorph of the known structure of $\mathrm{L} 1{ }^{\mathrm{Do}} \mathrm{AlMe}_{2}{ }^{[15]}$ and the bonding parameters (Al-O, Al-N and Al-C bond length, endo- and exocyclic bond angles) of both polymorphs are almost identical. In addition, the solid state structures of $\mathrm{L}^{\mathrm{Do} 1} \mathrm{Al}(\mathrm{Cl}) \mathrm{Me}$ (12) and $\mathrm{L}^{\mathrm{Do}{ }^{\circ 1}} \mathrm{AlCl}_{2}$ (13) are comparable to those of $\mathrm{L} 1^{\mathrm{Do} 2} \mathrm{Al}(\mathrm{Me}) \mathrm{Cl}^{[16]}$ and $\mathrm{L}^{1{ }^{\mathrm{Do} 2}} \mathrm{AlCl}_{2},{ }^{[17]}$ respectively, and their central Al-N (7 1.9857(7) $\AA$; 12 1.9658(11) $\AA ; 13$ 1.9414(6) $\AA ; 14$ 1.9719(8) $\AA$ ) and Al-O (7 1.8984(7) $\AA ; 12$ 1.8541(11) $\AA ; 13$ 1.8232(6) $\AA ; 14$ 1.8656(7) $\AA)$ bond lengths and O-Al-N bond angles $\left(788.65(3)^{\circ} ; 12\right.$ 89.92(5) $\left.1391.15(3)^{\circ} ; 1489.23(3)^{\circ}\right)$ within the six-membered $\mathrm{AlONC}_{3}$ metallacycle also agree very well. The $\mathrm{Al}$ atoms in the complexes are fivefold coordinated and show distorted trigonalbipyramidal (tbp) coordination modes. The central aluminum atom in 7 and $12-14$ is coordinated by $\mathrm{N} 1$ and two further heteroatoms $\mathrm{X}(\mathrm{X}=\mathrm{C}, \mathrm{Cl}, \mathrm{O})$ in a trigonal planar mode, whereas the $\mathrm{O} 1$ and N2 (amine) atoms always adopt the axial positions of the distorted tbp coordination. The O1-Al-N2 bond angles deviated from linearity by roughly $15^{\circ}$ (table 2 ).

Penta-coordinated aluminum complexes have been prepared and structurally characterized in the past and the bonding situation in such complexes including chelating tri- or tetradentate N,O-based ligands was analyzed by quantum chemical calculations. ${ }^{[18]}$ Lewiński et al. demonstrated the transeffect in five-coordinated aluminum complexes containing $\mathrm{O}, \mathrm{O}^{\prime}$ chelating substituents, clearly revealing the influence on the resulting structure in solution and in the solid state as well as the stability of such complexes. ${ }^{[19]}$ Several dimeric complexes of the general type $\left[\mathrm{R}_{2} \mathrm{Al}\left(\mathrm{O}, \mathrm{O}^{\prime}\right)\right]_{2}$ derived from $\alpha$ - and $\beta$-hydroxy carbonyl compounds were structurally characterized and a strong relation between the two axial $\mathrm{Al}-\mathrm{O}$ bond lengths was revealed: The stronger (shorter) the external axial $\mathrm{Al}-\mathrm{O}_{\mathrm{ax} / \mathrm{ex}}$ bond, the weaker (longer) the internal axial $\mathrm{Al}-\mathrm{O}_{\mathrm{ax} / \mathrm{br}}$ bond toward the bridging alkoxide. ${ }^{[20]}$ In addition, the formation of an envelopetype $\mathrm{C}_{3} \mathrm{O}_{2} \mathrm{Al}$ six-membered ring as observed in the 4methylpyridine adduct of $\mathrm{Me}_{2} \mathrm{Al}\left[\mathrm{OC}(\mathrm{Me}) \mathrm{C}_{6} \mathrm{H}_{4}-2-\mathrm{O}\right]$, which also contains a penta-coordinate aluminum atom, was addressed to the trans-effect of the axial substituents, even though no bond weakening was observed in this case. ${ }^{[7 b]}$ It should be noted, that the $\mathrm{C}_{3} \mathrm{NOAl}$ six memebered rings in 7 and 12-14 each form envelope-like structures.

The side-arm donor group $\left(\mathrm{NMe}_{2}\right)$ coordinates in all complexes to the Lewis-acidic aluminum atom, resulting in the formation of five-membered $\mathrm{AIN}_{2} \mathrm{C}_{2}$ rings, and the $\mathrm{Al}-\mathrm{N}$ bond lengths

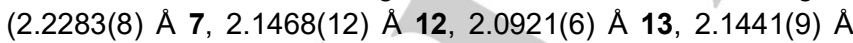
14) are comparable to those reported for $\mathrm{L} 1^{\mathrm{Do}} \mathrm{Al}(\mathrm{Me}) \mathrm{Cl}$ $(2.252(2) \AA)^{[16]}$ and $\mathrm{L}^{\mathrm{Do} o 2} \mathrm{AlCl}_{2}(2.171(3) \AA) \cdot{ }^{[17]}$ The axial Al-N2 are significantly elongated compared to the equatorial Al-N1 bond lengths. As was expected, the introduction of two electronegative, electron withdrawing $\mathrm{Cl}$ substituents increases the Lewis acidity of the central $\mathrm{Al}$ atom, resulting in the shortest Al-N bond distances.

Single crystals of $\mathrm{L} 1^{\mathrm{Do}} \mathrm{Ti}\left(\mathrm{Cp}^{*}\right) \mathrm{Me}_{2} 10$ were obtained upon storage of a concentrated solution of 10 in $n$-hexane at $-30{ }^{\circ} \mathrm{C} .{ }^{[11]}$ 10 adopts a monomeric structure in the solid state (Figure 7 ).

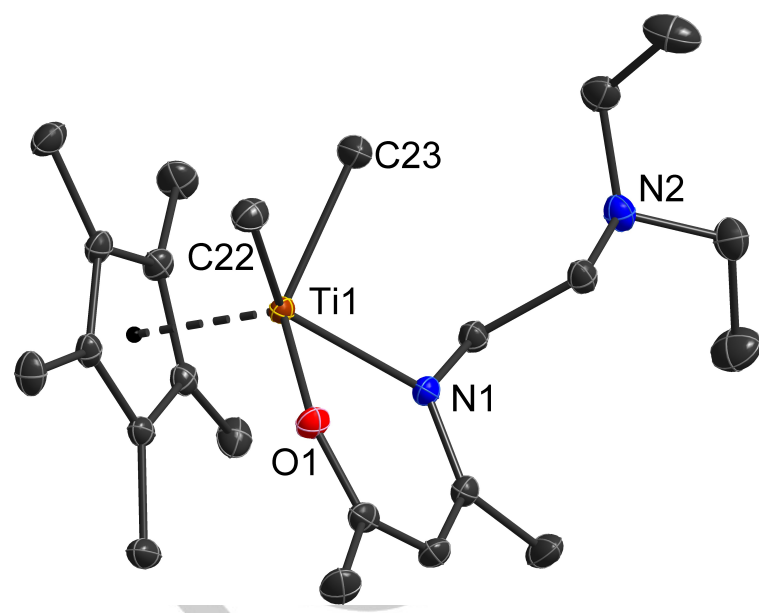

Figure 7. Crystal structure of $\mathrm{L} 1{ }^{\mathrm{Do} 2} \mathrm{Ti}\left(\mathrm{Cp}^{*}\right) \mathrm{Me}_{2}(10)$. All hydrogen atoms have been omitted for clarity. Ellipsoids are drawn to a $50 \%$ probability level.

Even though a large number of $\beta$-ketoiminate complexes of group 4 metal have been synthesized in the past,, ${ }^{[1]}$ only one compound containing a $\mathrm{Cp}^{*}$ substituent has been structurally characterized. The mixed ketoiminate-ketoimine zirconium complex $\mathrm{Cp}^{*}(\mathrm{Cl})_{2} \mathrm{Zr}(\mathrm{L} 5) \mathrm{L} 5 \mathrm{H}$, which contains a para- $\mathrm{CF}_{3}-$ substituted phenyl substituent at the imine $\mathrm{N}$ atom, was obtained from the reaction of $\mathrm{Cp}^{\star} \mathrm{ZrCl}_{3}$ with an equimolar mixture of LiL5 and $\mathrm{L} 5 \mathrm{H}\left(\mathrm{L} 5=\left\{\left(\mathrm{OC}(\mathrm{Me}) \mathrm{C}(\mathrm{H}) \mathrm{C}(\mathrm{Me}) \mathrm{N}-4-\mathrm{CF}_{3} \mathrm{Ph}\right)\right\}\right) .{ }^{[22]}$ The $\mathrm{Cp}^{*}$ substituent in 10 adopts a $\eta^{5}$ coordination mode as was expected form the ${ }^{1} \mathrm{H}$ and ${ }^{13} \mathrm{C}$ NMR spectra and as was also observed in $\mathrm{Cp}^{*}(\mathrm{Cl})_{2} \mathrm{Zr}(\mathrm{L} 5) \mathrm{L} 5 \mathrm{H}$. The Ti-O (1.963(2) $\AA$ ), Ti-N $(2.226(8) \AA)$ and Ti-Cp ${ }^{*}$ centr. bond lengths $(2.402(8) \AA)$ of 10 are shorter compared to the values observed for $\mathrm{Cp}^{*}(\mathrm{Cl})_{2} \mathrm{Zr}(\mathrm{L} 5) \mathrm{L} 5 \mathrm{H}$ ( $\mathrm{Zr}-\mathrm{O}(2.054(5) \AA), \mathrm{Zr}-\mathrm{N}(2.418(6) \AA)$ and $\mathrm{Zr}-\mathrm{Cp}^{*}{ }_{\text {centr }}(2.602(8) \AA)$ ), reflecting the larger atom radii of $\mathrm{Zr}$ compared to $\mathrm{Ti}$. The amine donor group in $\mathbf{1 0}$ does not bind to the Lewis-acidic metal center most likely due to the presence of the $\eta^{5}$-coordinated $\mathrm{Cp}^{*}$ ligand, which transfers enough electron density to the Ti atom. As a consequence, the $\mathrm{Ti}$ atom is fivefold coordinated and adopts a distorted square-pyramidal coordination geometry.

\section{Conclusions}

Metalation reactions of donor-functionalized $\beta$-ketoiminines L1$3^{\text {Do1-4 }} \mathrm{H}$ with main group $(\mathrm{Al})$ and transition metal complexes $(\mathrm{Ti}$, $\mathrm{Zr}, \mathrm{Zn}$ ) yielded heteroleptic metal $\beta$-ketoiminate complexes 1 15. The complexes were characterized by heteronuclear NMR $\left({ }^{1} \mathrm{H},{ }^{13} \mathrm{C}\right)$ and $\mathrm{IR}$ spectroscopy, elemental analysis as well as single crystal $X$-ray analyses $(2,4,7,10,12-14)$. Except for the heteroleptic $\mathrm{Ti}$ complex 10, the donor side-arm of the $\beta$ ketoimininate ligands was found to coordinate to the metal atom, resulting in the formation of monometallic complexes containing tetrahedrally-coordinated zinc and penta-coordinated aluminum atoms. 


\section{Experimental Section}

General: All manipulations involving air-sensitive materials were performed using standard vacuum techniques and inert gas atmosphere (argon, < 0.3 ppm $\mathrm{O}_{2}$ ) or in a glove box (MBraun Unilab V2.0). Solvents were carefully dried over $\mathrm{CaH}_{2}\left(\mathrm{CH}_{2} \mathrm{Cl}_{2}\right)$ or $\mathrm{Na} / \mathrm{K}$ alloy (pentane, hexane), respectively, degassed and stored in teflon-sealed glass vessel over molecular sieve. $\mathrm{Zn}\left[\mathrm{N}\left(\mathrm{SiMe}_{3}\right)_{2}\right]_{2}{ }^{[23]}$ and $\mathrm{Cp}^{*} \mathrm{MMe}_{3}(\mathrm{M}=\mathrm{Ti}, \mathrm{Zr})^{[24]}$ were synthesized according to literature methods. ${ }^{1} \mathrm{H}(300 \mathrm{MHz})$ and ${ }^{13} \mathrm{C}\{1 \mathrm{H}\}$ (75.5 MHz) NMR spectra $(\delta$ in $\mathrm{ppm}$ ) were recorded using a Bruker Avance DPX-300 spectrometer and the spectra were referenced to internal $\mathrm{C}_{6} \mathrm{D}_{5} \mathrm{H}\left({ }^{1} \mathrm{H}: \delta=7.16 ;{ }^{13} \mathrm{C}: \delta=128.06\right), \mathrm{C}_{6} \mathrm{D}_{5} \mathrm{CHD}_{2}\left({ }^{1} \mathrm{H}: \delta=2.08\right.$; $\left.{ }^{13} \mathrm{C}: \delta=20.43\right), \mathrm{CDCl}_{3}\left({ }^{1} \mathrm{H}: \delta=7.26 ;{ }^{13} \mathrm{C}: \delta=77.16\right)$ and $\mathrm{CD}_{2} \mathrm{Cl}_{2}\left({ }^{1} \mathrm{H}: \delta=\right.$ $\left.\left.5.32 ;{ }^{13} \mathrm{C}: \delta=53.84\right)\right) .{ }^{[25]}$ Microanalyses were performed at the Elemental Analysis Laboratory of the University of Duisburg-Essen. IR spectra were measured in an ALPHA-T FT-IR spectrometer equipped with a single reflection ATR sampling module. The melting points were measured using a Thermo Scientific 9300 apparatus.

General procedure for the synthesis of $\boldsymbol{\beta}$-ketoimines LH. A solution of the acetylacetone derivative in $15 \mathrm{~mL}$ of dichloromethane was cooled to $0{ }^{\circ} \mathrm{C}$, the amine dropwise added and the resulting solution stirred for additional $2 \mathrm{~h}$. The as-formed water as well as the solvent and the remaining acetylacetonate was removed in vacuo and the $\beta$-ketoimines were obtained as yellow oils.

L1 ${ }^{\text {Do3 }}$ H. Reagents: $6.2 \mathrm{~mL}$ (61 mmol) acetylacetone, $7.6 \mathrm{~mL}$ (60 mmol) 1(3-aminopropyl)pyrrolidine. Yield $11.01 \mathrm{~g}$ (93\%). Elemental analysis calcd (\%) for $\mathrm{C}_{11} \mathrm{H}_{20} \mathrm{~N}_{2} \mathrm{O}$ : C, 67.3; $\mathrm{H}, 10.3 ; \mathrm{N}, 14.3$; found (\%): C, 66.8, $\mathrm{H}$, 10.2, N, 14.4. ${ }^{1} \mathrm{H}-\mathrm{NMR}\left(300 \mathrm{MHz}, \mathrm{C}_{6} \mathrm{D}_{6}, 25^{\circ} \mathrm{C}\right): \delta=1.49\left(\mathrm{~s}, 3 \mathrm{H}, \mathrm{CCH}_{3}\right)$, $1.51\left(\mathrm{~m}, 4 \mathrm{H}, \mathrm{N}^{\text {pyr }} \mathrm{CH}_{2} \mathrm{CH}_{2}\right), 1.98\left(\mathrm{~s}, 3 \mathrm{H}, \mathrm{CCH}_{3}\right), 2.24\left(\mathrm{~m}, 4 \mathrm{H}, \mathrm{N}^{\text {pyr }} \mathrm{CH}_{2} \mathrm{CH}_{2}\right)$, $2.27\left(\mathrm{t},{ }^{3} \mathrm{~J}_{\mathrm{HH}}=6.6 \mathrm{~Hz}, 2 \mathrm{H}, \mathrm{NCH}_{2} \mathrm{CH}_{2}\right), 2.90\left(\mathrm{q},{ }^{3} \mathrm{~J}_{\mathrm{HH}}=5.8 \mathrm{~Hz}, 2 \mathrm{H}\right.$, $\left.\mathrm{NCH}_{2} \mathrm{CH}_{2}\right), 4.88$ (s, $\left.1 \mathrm{H}, \mathrm{CH}\right), 11.13(\mathrm{~s}, 1 \mathrm{H}, \mathrm{NH}) .{ }^{13} \mathrm{C} \mathrm{NMR}\left(75 \mathrm{MHz}, \mathrm{C}_{6} \mathrm{D}_{6}\right.$, $\left.25{ }^{\circ} \mathrm{C}\right): \delta=18.6\left(\mathrm{CCH}_{3}\right), 23.9\left(\mathrm{~N}^{\mathrm{pyr}} \mathrm{CH}_{2} \mathrm{CH}_{2}\right), 28.9\left(\mathrm{CCH}_{3}\right), 42.3$ $\left(\mathrm{NCH}_{2} \mathrm{CH}_{2}\right), 54.2\left(\mathrm{~N}^{\text {pyr }} \mathrm{CH}_{2} \mathrm{CH}_{2}\right), 55.8\left(\mathrm{NCH}_{2} \mathrm{CH}_{2}\right), 96.2(\mathrm{CH}), 161.6(\mathrm{CN})$, 193.9 (CO). IR: $v=3025,2955,2889,2804,1609,1546,1519,1297$, $1174,1122,1010,712,633 \mathrm{~cm}^{-1}$

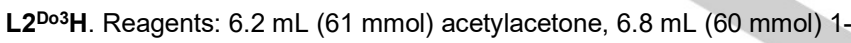
(3-aminopropyl)pyrrolidine. Yield $12.15 \mathrm{~g}$ (96\%). Elemental analysis calcd (\%) for $\mathrm{C}_{12} \mathrm{H}_{22} \mathrm{~N}_{2} \mathrm{O}$ : C, 68.5; $\mathrm{H}, 10.5 ; \mathrm{N}, 13.3$; found (\%): C, 69.0, $\mathrm{H}$, 10.6, N, 13.2. ${ }^{1} \mathrm{H}-\mathrm{NMR}\left(300 \mathrm{MHz}, \mathrm{C}_{6} \mathrm{D}_{6}, 25^{\circ} \mathrm{C}\right): \delta=1.38\left(\mathrm{~m},{ }^{3} \mathrm{~J}_{\mathrm{HH}}=6.8\right.$ $\left.\mathrm{Hz}, 2 \mathrm{H}, \mathrm{NCH}_{2} \mathrm{CH}_{2} \mathrm{CH}_{2}\right), 1.53\left(\mathrm{~s}, 3 \mathrm{H}, \mathrm{CCH}_{3}\right), 1.55\left(\mathrm{~m},{ }^{3} \mathrm{~J}_{\mathrm{HH}}=3.6 \mathrm{~Hz}, 4 \mathrm{H}\right.$, $\left.\mathrm{N}^{\text {pyr }} \mathrm{CH}_{2} \mathrm{CH}_{2}\right), 1.96\left(\mathrm{~s}, 3 \mathrm{H}, \mathrm{CCH}_{3}\right), 2.24\left(\mathrm{t},{ }^{3} \mathrm{~J}_{\mathrm{HH}}=6.6 \mathrm{~Hz}, 6 \mathrm{H}, \mathrm{N}^{\text {pyr }} \mathrm{CH}_{2}\right)$, $2.92\left(\mathrm{q},{ }^{3} \mathrm{~J}_{\mathrm{HH}}=5.8 \mathrm{~Hz}, 2 \mathrm{H}, \mathrm{NCH}_{2} \mathrm{CH}_{2} \mathrm{CH}_{2}\right), 4.85(\mathrm{~s}, 1 \mathrm{H}, \mathrm{CH}), 11.10(\mathrm{~s}, 1 \mathrm{H}$, $\mathrm{NH}) .{ }^{13} \mathrm{C}$ NMR $\left(75 \mathrm{MHz}, \mathrm{C}_{6} \mathrm{D}_{6}, 25{ }^{\circ} \mathrm{C}\right): \delta=18.0\left(\mathrm{CCH}_{3}\right), 23.9$ $\left(\mathrm{N}^{\text {pyr }} \mathrm{CH}_{2} \mathrm{CH}_{2}\right), 28.9\left(\mathrm{CCH}_{3}\right), 29.6\left(\mathrm{NCH}_{2} \mathrm{CH}_{2} \mathrm{CH}_{2}\right), 40.7\left(\mathrm{NCH}_{2} \mathrm{CH}_{2} \mathrm{CH}_{2}\right)$, $53.0\left(\mathrm{~N}^{\text {pyr }} \mathrm{CH}_{2} \mathrm{CH}_{2}\right), 54.1\left(\mathrm{NCH}_{2} \mathrm{CH}_{2} \mathrm{CH}_{2}\right), 95.1(\mathrm{CH}), 162.2(\mathrm{CN}), 193.7$ (CO). IR: $v=2974,2952,2831,2822,2740,1638,1574,1423,1307$, $1089,1062,795,637 \mathrm{~cm}^{-1}$.

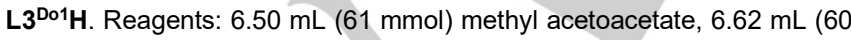
mmol) N,N-dimethylethylenediamine. Yield $10.84 \mathrm{~g}(97 \%)$. Elemental analysis calcd (\%) for $\mathrm{C}_{9} \mathrm{H}_{18} \mathrm{~N}_{2} \mathrm{O}_{2}$ : C, 58.0; $\mathrm{H}, 9.7 ; \mathrm{N}, 15.0$; found (\%): C, 57.7; $\mathrm{H}, 10.0 ; \mathrm{N}, 15.0 .{ }^{1} \mathrm{H}-\mathrm{NMR}\left(300 \mathrm{MHz}, \mathrm{CDCl}_{3}, 25^{\circ} \mathrm{C}\right): \delta=1.85(\mathrm{~s}, 3 \mathrm{H}$ $\left.\mathrm{CCH}_{3}\right), 2.18\left(\mathrm{~s}, 6 \mathrm{H}, \mathrm{N}\left(\mathrm{CH}_{3}\right)_{2}\right), 2.39\left(\mathrm{~m}, 2 \mathrm{H}, \mathrm{NCH}_{2} \mathrm{CH}_{2}\right), 3.20(\mathrm{~m}, 2 \mathrm{H}$, $\left.\mathrm{NCH}_{2} \mathrm{CH}_{2}\right), 3.53\left(\mathrm{~s}, 3 \mathrm{H}, \mathrm{OCH}_{3}\right), 4.36(\mathrm{~s}, 1 \mathrm{H}, \mathrm{CH}), 8.55(\mathrm{~s}, 1 \mathrm{H}, \mathrm{NH}) .{ }^{13} \mathrm{C}-$ NMR $\left(75 \mathrm{MHz}, \mathrm{CDCl}_{3}, 25{ }^{\circ} \mathrm{C}\right): \delta=19.6\left(\mathrm{CCH}_{3}\right), 41.0\left(\mathrm{NCH}_{2} \mathrm{CH}_{2}\right), 45.5$ $\left(\mathrm{N}\left(\mathrm{CH}_{3}\right)_{2}\right), 49.9\left(\mathrm{OCH}_{3}\right), 59.0\left(\mathrm{NCH}_{2} \mathrm{CH}_{2}\right), 81.9(\mathrm{CH}), 161.5(\mathrm{CN}), 170.7$ (CO). IR: $v=3354,3266,2945,2861,2820,2768,1654,1593,1499$, $1443,1242,1168,1119,1051,920,782,699 \mathrm{~cm}^{-1}$.
L3 ${ }^{\text {Do4 }} \mathrm{H}$. Reagents: $6.50 \mathrm{~mL}(61 \mathrm{mmol})$ methyl acetoacetate, $4.78 \mathrm{~g}$ (60 mmol) 2-methoxyethylamine. Yield $7.63 \mathrm{~g}(96 \%)$. Elemental analysis calcd (\%) for $\mathrm{C}_{8} \mathrm{H}_{15} \mathrm{NO}_{3}$ : C, 55.5; $\mathrm{H}, 8.7 ; \mathrm{N}, 8.1$; found (\%): C, 55.9; $\mathrm{H}$, 8.9; $\mathrm{N}, 7.8 .{ }^{1} \mathrm{H}-\mathrm{NMR}\left(300 \mathrm{MHz}, \mathrm{CDCl}_{3}, 25^{\circ} \mathrm{C}\right): \delta=1.89$ (s, $\left.3 \mathrm{H}, \mathrm{CCH}_{3}\right)$, $3.34\left(\mathrm{~s}, 3 \mathrm{H}, \mathrm{OCH}_{3}\right), 3.44\left(\mathrm{dt},{ }^{3} \mathrm{JHH}_{\mathrm{HH}}=5.6 \mathrm{~Hz}, 2 \mathrm{H}, \mathrm{NCH}_{2} \mathrm{CH}_{2}\right), 3.47\left(\mathrm{t},{ }^{3} \mathrm{~J}_{\mathrm{HH}}=\right.$ $\left.5.6 \mathrm{~Hz}, 2 \mathrm{H}, \mathrm{NCH}_{2} \mathrm{CH}_{2}\right), 3.57$ (s, 3H, $\left.\mathrm{COCH}_{3}\right), 4.42(\mathrm{~s}, 1 \mathrm{H}, \mathrm{CH}), 8.62$ (s, $1 \mathrm{H}, \quad \mathrm{NH}) .{ }^{13} \mathrm{C}-\mathrm{NMR}\left(75 \mathrm{MHz}, \mathrm{CDCl}_{3}, 25{ }^{\circ} \mathrm{C}\right): 19.5\left(\mathrm{CCH}_{3}\right), 42.9$ $\left(\mathrm{NCH}_{2} \mathrm{CH}_{2}\right), 49.9\left(\mathrm{OCH}_{3}\right), 59.1\left(\mathrm{NCH}_{2} \mathrm{CH}_{2}\right), 71.9\left(\mathrm{COCH}_{3}\right), 82.3(\mathrm{CH})$ 161.8 (CN), 170.9 (CO). IR: $v=3353,3291,2929,2880,2833,1652$, $1598,1502,1443,1257,1170,1115,1055,1003,923,782,698 \mathrm{~cm}^{-1}$

General procedure for the synthesis of heteroleptic complexes $\mathrm{LZn}\left(\mathrm{N}\left(\mathrm{SiMe}_{3}\right)_{2}\right.$ : A solution of the $\beta$-ketoimine in $n$-hexane $(20 \mathrm{~mL})$ was slowly added to a cooled $\left(-50{ }^{\circ} \mathrm{C}\right)$ solution of $\mathrm{Zn}\left[\mathrm{N}\left(\mathrm{SiMe}_{3}\right)_{2}\right]_{2}(0.33 \mathrm{~g}, 0.84$ $\mathrm{mmol})$ in $n$-hexane $(20 \mathrm{~mL})$ and slowly warmed to ambient temperature within $3 \mathrm{~h}$. The solution was concentrated to $5 \mathrm{~mL}$ and stored at $-30{ }^{\circ} \mathrm{C}$, yielding colorless crystalline solids within $24 \mathrm{~h}$.

L1 ${ }^{\text {Do1 }} \mathrm{ZnN}\left(\mathrm{SiMe}_{3}\right)_{2}$ 1: Reagent: $0.14 \mathrm{~g}(0.84 \mathrm{mmol})$ L1 ${ }^{\mathrm{Do1}} \mathrm{H}$. Yield $0.29 \mathrm{~g}$ (82\%). Mp $87^{\circ} \mathrm{C}$. Elemental analysis calcd (\%) for $\mathrm{C}_{15} \mathrm{H}_{35} \mathrm{~N}_{3} \mathrm{OSi} 2 \mathrm{Zn}$ : C 45.6, H 8.9, N 10.6; found (\%): C 45.2, H 8.8, N 10.4. ${ }^{1} \mathrm{H} \mathrm{NMR} \mathrm{(300} \mathrm{MHz,}$ $\mathrm{CD}_{2} \mathrm{Cl}_{2}, 25^{\circ} \mathrm{C}$ ): $\delta=0.01\left(\mathrm{~s}, 18 \mathrm{H}, \mathrm{SiMe}_{3}\right), 1.87\left(\mathrm{~s}, 6 \mathrm{H}, \mathrm{CCH}_{3}\right), 2.39(\mathrm{~s}, 6 \mathrm{H}$ $\left.\mathrm{N}\left(\mathrm{CH}_{3}\right)_{2}\right), 2.64\left(\mathrm{t},{ }^{3} \mathrm{~J}_{\mathrm{HH}}=6.3 \mathrm{~Hz}, 2 \mathrm{H}, \mathrm{NCH}_{2} \mathrm{CH}_{2}\right), 3.39\left(\mathrm{t},{ }^{3} \mathrm{~J}_{\mathrm{HH}}=6.3 \mathrm{~Hz}, 2 \mathrm{H}\right.$, $\left.\mathrm{NCH}_{2} \mathrm{CH}_{2}\right), 4.48(\mathrm{~s}, 1 \mathrm{H}, \mathrm{CH}) \cdot{ }^{13} \mathrm{C}$ NMR $\left(75 \mathrm{MHz}, \mathrm{CD}_{2} \mathrm{Cl}_{2}, 25{ }^{\circ} \mathrm{C}\right): \delta=5.0$ $\left(\mathrm{SiMe}_{3}\right), 21.2\left(\mathrm{CCH}_{3}\right), 27.6\left(\mathrm{CCH}_{3}\right), 44.0\left(\mathrm{NCH}_{2} \mathrm{CH}_{2}\right), 45.4\left(\mathrm{~N}\left(\mathrm{CH}_{3}\right)_{2}\right)$, $58.8\left(\mathrm{NCH}_{2} \mathrm{CH}_{2}\right), 96.9(\mathrm{CH}), 172.3(\mathrm{CN}), 182.9(\mathrm{CO}) . \mathrm{IR}: \mathrm{v} 2912,1584$ $1509,1396,1281,1067,1000,948,799,675,552,464,420$

L1 ${ }^{\mathrm{Do} 2} \mathrm{ZnN}\left(\mathrm{SiMe}_{3}\right)_{2}$ 2: Reagent: $0.17 \mathrm{~g}(0.84 \mathrm{mmol})$ L1 ${ }^{\mathrm{Do}} \mathrm{H}$. Yield $0.31 \mathrm{~g}$ (86\%). Mp $31^{\circ} \mathrm{C}$. Elemental analysis calcd (\%) for $\mathrm{C}_{17} \mathrm{H}_{39} \mathrm{~N}_{3} \mathrm{OSi} 2 \mathrm{Zn}$ : C 48.3, H 9.3, N 9.9; found (\%): C 47.9, H 9.2, N 9.8. ${ }^{1} \mathrm{H}$ NMR (300 MHz, $\left.\mathrm{C}_{6} \mathrm{D}_{6}, 25{ }^{\circ} \mathrm{C}\right): \delta=0.43\left(\mathrm{~s}, 18 \mathrm{H}, \mathrm{SiMe}_{3}\right), 0.79\left(\mathrm{t},{ }^{3} J_{\mathrm{HH}}=7.2 \mathrm{~Hz}, 6 \mathrm{H}\right.$, $\left.\mathrm{N}\left(\mathrm{CH}_{2} \mathrm{CH}_{3}\right)_{2}\right), 1.44\left(\mathrm{~s}, 3 \mathrm{H}, \mathrm{CCH}_{3}\right), 2.00\left(\mathrm{~s}, 3 \mathrm{H}, \mathrm{CCH}_{3}\right), 2.20\left(\mathrm{t},{ }^{3} \mathrm{~J}_{\mathrm{HH}}=6.4\right.$ $\mathrm{Hz}, 2 \mathrm{H}, \mathrm{NCH}_{2} \mathrm{CH}_{2}$ ), 2.39 (q, $\left.{ }^{3} \mathrm{~J}_{\mathrm{HH}}=7.2 \mathrm{~Hz}, 4 \mathrm{H}, \mathrm{N}\left(\mathrm{CH}_{2} \mathrm{CH}_{3}\right)_{2}\right), 2.81$ (t, ${ }^{3} \mathrm{~J}_{\mathrm{HH}}$ $\left.=6.4 \mathrm{~Hz}, 2 \mathrm{H}, \mathrm{NCH}_{2} \mathrm{CH}_{2}\right), 4.84(\mathrm{~s}, 1 \mathrm{H}, \mathrm{CH}) .{ }^{13} \mathrm{C} \mathrm{NMR}\left(75 \mathrm{MHz}, \mathrm{C}_{6} \mathrm{D}_{6}\right.$ $\left.25{ }^{\circ} \mathrm{C}\right): \delta=6.2\left(\mathrm{SiMe}_{3}\right), 9.6\left(\mathrm{~N}\left(\mathrm{CH}_{2} \mathrm{CH}_{3}\right)_{2}\right), 21.3\left(\mathrm{CCH}_{3}\right), 28.4\left(\mathrm{CCH}_{3}\right)$, $43.7\left(\mathrm{NCH}_{2} \mathrm{CH}_{2}\right), 45.6\left(\mathrm{~N}\left(\mathrm{CH}_{2} \mathrm{CH}_{3}\right)_{2}\right), 52.3\left(\mathrm{NCH}_{2} \mathrm{CH}_{2}\right), 97.7(\mathrm{CH}), 172.0$ (CN), 184.0 (CO). IR: v 2945, 1595, 1499, 1387, 1342, 1241, 992, 880, $815,740,664,610,439,403$

General procedure for the synthesis of heteroleptic complexes LZnEt. $10 \mathrm{~mL}(10 \mathrm{mmol})$ of a $1 \mathrm{M}$ solution of $\mathrm{Et}_{2} \mathrm{Zn}$ in $n$-hexane was cooled to $-35{ }^{\circ} \mathrm{C}$ and a solution of the corresponding $\beta$-ketoimine in 25 $\mathrm{mL}$ of $n$-hexane was added dropwise. The solution was slowly warmed to ambient temperature and stirred for additional $2 \mathrm{~h}$, until ethane evolution has stopped. The solvent was removed in vacuo and the remaining crystalline solid recrystallized from solutions in $n$-hexane at $-30{ }^{\circ} \mathrm{C}$. The complexes were obtained as light-yellow crystals.

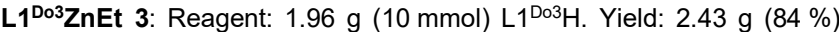
$\mathrm{Mp} 83{ }^{\circ} \mathrm{C}$. Elemental analysis calcd (\%) for $\mathrm{C}_{13} \mathrm{H}_{24} \mathrm{~N}_{2} \mathrm{OZn}$ : C, 53.9; $\mathrm{H}, 8.4$ $\mathrm{N}, 9.7$; found (\%): C, 54.0; $\mathrm{H}, 8.3 ; \mathrm{N}, 9.9 .{ }^{1} \mathrm{H}$ NMR $\left(300 \mathrm{MHz}, \mathrm{C}_{6} \mathrm{D}_{6}\right.$, $\left.25{ }^{\circ} \mathrm{C}\right): \delta=0.63\left(\mathrm{q},{ }^{3} \mathrm{~J}_{\mathrm{HH}}=7.9 \mathrm{~Hz}, 2 \mathrm{H}, \mathrm{ZnCH}_{2} \mathrm{CH}_{3}\right), 1.61(\mathrm{~m}, 4 \mathrm{H}$ $\mathrm{N}^{\text {pyr }} \mathrm{CH}_{2} \mathrm{CH}_{2}$ ), 1.62 (s, 3H, $\left.\mathrm{CCH}_{3}\right), 1.81\left(\mathrm{t},{ }^{3} \mathrm{~J}_{\mathrm{HH}}=7.9 \mathrm{~Hz}, 3 \mathrm{H}, \mathrm{ZnCH}_{2} \mathrm{CH}_{3}\right)$, $2.14\left(\mathrm{~s}, 3 \mathrm{H}, \mathrm{CCH}_{3}\right), 2.30\left(\mathrm{~m}, 6 \mathrm{H}, \mathrm{N}^{\text {pyr }} \mathrm{CH}_{2} \mathrm{CH}_{2}\right.$ and $\left.\mathrm{NCH}_{2} \mathrm{CH}_{2}\right), 2.91(\mathrm{t}$ $\left.{ }^{3} \mathrm{~J}_{\mathrm{HH}}=6.3 \mathrm{~Hz}, 2 \mathrm{H}, \mathrm{NCH}_{2} \mathrm{CH}_{2}\right), 4.97(\mathrm{~s}, 1 \mathrm{H}, \mathrm{CH}) .{ }^{13} \mathrm{C} \mathrm{NMR}\left(75 \mathrm{MHz}, \mathrm{C}_{6} \mathrm{D}_{6}\right.$, $\left.25{ }^{\circ} \mathrm{C}\right): \delta=-2.8\left(\mathrm{ZnCH}_{2} \mathrm{CH}_{3}\right), 13.5\left(\mathrm{ZnCH}_{2} \mathrm{CH}_{3}\right), 20.6\left(\mathrm{CCH}_{3}\right), 22.7$ $\left(\mathrm{N}^{\text {pyr }} \mathrm{CH}_{2} \mathrm{CH}_{2}\right), 27.6\left(\mathrm{CCH}_{3}\right), 45.5\left(\mathrm{NCH}_{2} \mathrm{CH}_{2}\right), 52.8\left(\mathrm{~N}^{\text {pyr }} \mathrm{CH}_{2} \mathrm{CH}_{2}\right), 54.0$ $\left(\mathrm{NCH}_{2} \mathrm{CH}_{2}\right), 96.9(\mathrm{CH}), 170.4(\mathrm{CN}), 182.3(\mathrm{CO}) . \mathrm{IR}: \mathrm{v}=2922,2851,1560$, $1506,1405,1310,1006,937,753,594,494,434 \mathrm{~cm}^{-1}$.

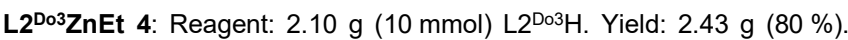
Mp $62{ }^{\circ} \mathrm{C}$. Elemental analysis calcd (\%) for $\mathrm{C}_{14} \mathrm{H}_{26} \mathrm{~N}_{2} \mathrm{OZn}$ : C, 55.4; $\mathrm{H}, 8.6$ 
$\mathrm{N}, 9.2 ;$ found (\%): C, 55.9; $\mathrm{H}, 8.8 ; \mathrm{N}, 9.3 .{ }^{1} \mathrm{H}$ NMR $\left(300 \mathrm{MHz}, \mathrm{C}_{6} \mathrm{D}_{6}\right.$ $\left.25{ }^{\circ} \mathrm{C}\right): \delta=0.54\left(\mathrm{q},{ }^{3} \mathrm{~J}_{\mathrm{HH}}=7.8 \mathrm{~Hz}, 2 \mathrm{H}, \mathrm{ZnCH}_{2} \mathrm{CH}_{3}\right), 1.16\left(\mathrm{~m},{ }^{3} \mathrm{~J}_{\mathrm{HH}}=4.8\right.$ $\left.\mathrm{Hz}, 4 \mathrm{H}, \mathrm{NCH}_{2} \mathrm{CH}_{2} \mathrm{CH}_{2}\right), 1.52\left(\mathrm{~s}, 3 \mathrm{H}, \mathrm{CCH}_{3}\right), 1.64\left(\mathrm{~m},{ }^{3} \mathrm{JHH}_{\mathrm{HH}}=5.9 \mathrm{~Hz}\right.$, $\left.\mathrm{N}^{\text {pyr }} \mathrm{CH}_{2} \mathrm{CH}_{2}\right), 1.71\left(\mathrm{t},{ }^{3} \mathrm{JHH}_{\mathrm{HH}}=7.9 \mathrm{~Hz}, 3 \mathrm{H}, \mathrm{ZnCH}_{2} \mathrm{CH}_{3}\right), 1.98\left(\mathrm{~s}, 3 \mathrm{H}, \mathrm{CCH}_{3}\right)$, $2.18\left(\mathrm{t},{ }^{3} \mathrm{JHH}_{\mathrm{HH}}=6.2 \mathrm{~Hz}, 4 \mathrm{H}, \mathrm{N}^{\text {pyr }} \mathrm{CH}_{2} \mathrm{CH}_{2}\right), 2.31\left(\mathrm{t},{ }^{3} \mathrm{JHH}_{\mathrm{HH}}=6.0 \mathrm{~Hz}, 2 \mathrm{H}\right.$, $\mathrm{NCH}_{2} \mathrm{CH}_{2} \mathrm{CH}_{2}$ ), 3.20 (t, $\left.{ }^{3} \mathrm{H}_{\mathrm{HH}}=4.5 \mathrm{~Hz}, 2 \mathrm{H}, \mathrm{NCH}_{2} \mathrm{CH}_{2} \mathrm{CH}_{2}\right), 4.67(\mathrm{~s}, 1 \mathrm{H}$ $\mathrm{CH}) .{ }^{13} \mathrm{C}$ NMR $\left(75 \mathrm{MHz}, \mathrm{CD}_{2} \mathrm{Cl}_{2}, 25{ }^{\circ} \mathrm{C}\right): \delta=-4.2\left(\mathrm{ZnCH}_{2} \mathrm{CH}_{3}\right), 14.2$ $\left(\mathrm{ZnCH}_{2} \mathrm{CH}_{3}\right), \quad 19.3\left(\mathrm{CCH}_{3}\right), 22.6\left(\mathrm{~N}^{\text {pyr }} \mathrm{CH}_{2} \mathrm{CH}_{2}\right), 27.7\left(\mathrm{CCH}_{3}\right), 29.0$ $\left(\mathrm{NCH}_{2} \mathrm{CH}_{2} \mathrm{CH}_{2}\right), \quad 50.4 \quad\left(\mathrm{NCH}_{2} \mathrm{CH}_{2} \mathrm{CH}_{2}\right), \quad 55.0 \quad\left(\mathrm{~N}^{\text {pyr }} \mathrm{CH}_{2} \mathrm{CH}_{2}\right), \quad 58.0$ $\left(\mathrm{NCH}_{2} \mathrm{CH}_{2} \mathrm{CH}_{2}\right), 96.1(\mathrm{CH}), 167.9(\mathrm{CN}), 181.7(\mathrm{CO}) . \mathrm{IR}: v=2905,2858$, $1545,1499,1422,1307,1036,978,596,565,471,432 \mathrm{~cm}^{-1}$.

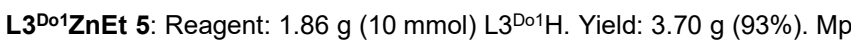
$80{ }^{\circ} \mathrm{C}$. Elemental analysis calcd (\%) for $\mathrm{C}_{11} \mathrm{H}_{22} \mathrm{~N}_{2} \mathrm{O}_{2} \mathrm{Zn}: \mathrm{C}, 47.2 ; \mathrm{H}, 7.9 ; \mathrm{N}$, 10.0; found (\%): C, 47.3; $\mathrm{H}, 7.8 ; \mathrm{N}, 10.0$. ${ }^{1} \mathrm{H}-\mathrm{NMR}\left(300 \mathrm{MHz}, \mathrm{CDCl}_{3}\right.$, $\left.25^{\circ} \mathrm{C}\right): \delta=0.13\left(\mathrm{q},{ }^{3} \mathrm{JHH}=8.02 \mathrm{~Hz}, 2 \mathrm{H}, \mathrm{ZnCH}_{2} \mathrm{CH}_{3}\right), 1.22\left(\mathrm{t},{ }^{3} \mathrm{JHH}_{\mathrm{HH}}=8.2\right.$ $\left.3 \mathrm{H}, \mathrm{ZnCH}_{2} \mathrm{CH}_{3}\right), 1.92\left(\mathrm{~s}, 3 \mathrm{H}, \mathrm{CCH}_{3}\right), 2.38\left(\mathrm{~s}, 6 \mathrm{H}, \mathrm{N}\left(\mathrm{CH}_{3}\right)_{2}\right), 2.57\left(\mathrm{t},{ }^{3} \mathrm{JHH}_{\mathrm{HH}}=\right.$ $\left.6.1 \mathrm{~Hz}, 2 \mathrm{H}, \mathrm{NCH}_{2} \mathrm{CH}_{2}\right), 3.39\left(\mathrm{t},{ }^{3} \mathrm{JHH}_{\mathrm{HH}}=5.8 \mathrm{~Hz}, 2 \mathrm{H}, \mathrm{NCH}_{2} \mathrm{CH}_{2}\right), 3.65(\mathrm{~s}, 3 \mathrm{H}$ $\left.\mathrm{OCH}_{3}\right), 4.33(\mathrm{~s}, 1 \mathrm{H}, \mathrm{CH}) \cdot{ }^{13} \mathrm{C}-\mathrm{NMR}\left(75 \mathrm{MHz}, \mathrm{CDCl}_{3}, 25^{\circ} \mathrm{C}\right): \delta=-2.3$ $\left(\mathrm{ZnCH}_{2} \mathrm{CH}_{3}\right), 1.2\left(\mathrm{ZnCH}_{2} \mathrm{CH}_{3}\right), 12.8\left(\mathrm{CCH}_{3}\right), 45.1\left(\mathrm{NCH}_{2} \mathrm{CH}_{2}\right), 47.4$ $\left(\mathrm{N}\left(\mathrm{CH}_{3}\right)_{2}\right), 50.7\left(\mathrm{OCH}_{3}\right), 58.9\left(\mathrm{NCH}_{2} \mathrm{CH}_{2}\right), 78.0(\mathrm{CH}), 129.2(\mathrm{CN}), 172.0$ (CO). IR: $v=2925,2873,2844,2801,1561,1516,1438,1367,1326$, $1286,1179,1065,1025,957,883,773,598,542,502,410 \mathrm{~cm}^{-1}$.

L3 ${ }^{\text {Do4 }}$ ZnEt 6: Reagent: $1.63 \mathrm{~g}$ (10 mmol) L3 ${ }^{\mathrm{D} 04} \mathrm{H}$. Yield: $2.88 \mathrm{~g}$ (72\%). Mp. $73{ }^{\circ} \mathrm{C}$. Elemental analysis calcd (\%) for $\mathrm{C}_{10} \mathrm{H}_{19} \mathrm{NO}_{3} \mathrm{Zn}: \mathrm{C}, 45.1 ; \mathrm{H}, 7.2 ; \mathrm{N}$ 5.3; found (\%): C, 44.7; $\mathrm{H}, 7.1 ; \mathrm{N}, 5.1 .{ }^{1} \mathrm{H}-\mathrm{NMR}\left(300 \mathrm{MHz}, \mathrm{CDCl}_{3}, 25^{\circ} \mathrm{C}\right)$ : $\delta=0.29\left(\mathrm{q},{ }^{3} \mathrm{JHH}_{\mathrm{HH}}=8.13 \mathrm{~Hz}, 2 \mathrm{H}, \mathrm{ZnCH}_{2} \mathrm{CH}_{3}\right), 1.19\left(\mathrm{t},{ }^{3} \mathrm{JHH}_{\mathrm{HH}}=8.2,3 \mathrm{H}\right.$ $\mathrm{ZnCH}_{2} \mathrm{CH}_{3}$ ), 1.96 (s, 3H, $\mathrm{CCH}_{3}$ ), 3.31 (s (br), 3H, OCH $\mathrm{OCH}_{3}, 3.50$ (s (br), $4 \mathrm{H}$ $\left.\mathrm{NCH}_{2} \mathrm{CH}_{2}\right), 3.63\left(\mathrm{~s}, 3 \mathrm{H}, \mathrm{COCH}_{3}\right), 4.37\left(\mathrm{~s}, 1 \mathrm{H}, \mathrm{C}_{\gamma} \mathrm{H}\right) .{ }^{13} \mathrm{C}-\mathrm{NMR}(75 \mathrm{MHz}$ $\left.\mathrm{CDCl}_{3}, 25^{\circ} \mathrm{C}\right): \delta=0.3\left(\mathrm{ZnCH}_{2} \mathrm{CH}_{3}\right), 1.1\left(\mathrm{ZnCH}_{2} \mathrm{CH}_{3}\right), 12.0\left(\mathrm{CCH}_{3}\right), 22.8$ $\left(\mathrm{OCH}_{3}\right), 49.8\left(\mathrm{NCH}_{2} \mathrm{CH}_{2}\right), 51.4\left(\mathrm{NCH}_{2} \mathrm{CH}_{2}\right), 59.0\left(\mathrm{COCH}_{3}\right), 72.3(\mathrm{CH})$, 126.4 (CN), 172.4 (CO). IR: $v=2939,2876,2853,1422,1287,1259$ $1203,1130,1059,993,906,769,607,513,446 \mathrm{~cm}^{-1}$.

Synthesis of heteroleptic complexes LAIMe ${ }_{2}$ : A solution of $L 1^{D 01} \mathrm{H}$ $(2.72 \mathrm{~g}, 16 \mathrm{mmoL})$ in $n$-pentane $(20 \mathrm{~mL})$ was slowly added to a solution of $\mathrm{AlMe}_{3}(1.64 \mathrm{~mL}, 94 \%, 16 \mathrm{mmoL})$ in $20 \mathrm{~mL}$ of $n$-pentane at $0{ }^{\circ} \mathrm{C}$, and the mixture was stirred for $5 \mathrm{~h}$ at ambient temperature. The solvent was removed in vacuo, yielding yellow waxy solids, which were recrystallized from solutions in $n$-pentane at $-80^{\circ} \mathrm{C}$. Light yellow crystalline solids were formed after storage for $24 \mathrm{~h}$.

L1 ${ }^{\text {Do1 }} \mathrm{AlMe}_{2}$ 7: Reagent: $2.72 \mathrm{~g}(16 \mathrm{mmoL}) \mathrm{L}^{\mathrm{Do}}{ }^{\mathrm{H}} \mathrm{H}$. Yield $3.40 \mathrm{~g}(94 \%)$. Mp $62{ }^{\circ} \mathrm{C}$. Elemental analysis calcd (\%) for $\mathrm{C}_{11} \mathrm{H}_{23} \mathrm{~N}_{2} \mathrm{OAl}$ : C 58.4, $\mathrm{H} 10.2$, $\mathrm{N}$ 12.4\%; found (\%): C 58.1, H 10.2, N 12.2. ${ }^{1} \mathrm{H}$ NMR (300 MHz, $\mathrm{C}_{6} \mathrm{D}_{6}$, $\left.25{ }^{\circ} \mathrm{C}\right): \delta=-0.32\left(\mathrm{~s}, 6 \mathrm{H}, \mathrm{Al}\left(\mathrm{CH}_{3}\right)_{2}\right), 1.35\left(\mathrm{~s}, 3 \mathrm{H}, \mathrm{CCH}_{3}\right), 1.81(\mathrm{~s}, 6 \mathrm{H}$, $\left.\mathrm{N}\left(\mathrm{CH}_{3}\right)_{2}\right), 1.86\left(\mathrm{~s}, 3 \mathrm{H}, \mathrm{CCH}_{3}\right), 2.10\left(\mathrm{t},{ }^{3} \mathrm{JHH}_{\mathrm{HH}}=6.6 \mathrm{~Hz}, 2 \mathrm{H}, \mathrm{NCH}_{2} \mathrm{CH}_{2}\right), 2.55$ $\left(\mathrm{t},{ }^{3} \mathrm{JHH}_{\mathrm{HH}}=6.5 \mathrm{~Hz}, 2 \mathrm{H}, \mathrm{NCH}_{2} \mathrm{CH}_{2}\right), 4.84(\mathrm{~s}, 1 \mathrm{H}, \mathrm{CH}) \cdot{ }^{13} \mathrm{C} \mathrm{NMR}(75 \mathrm{MHz}$, $\left.\mathrm{C}_{6} \mathrm{D}_{6}, 25{ }^{\circ} \mathrm{C}\right): \delta=-7.9\left(\mathrm{Al}\left(\mathrm{CH}_{3}\right)_{2}\right), 21.9\left(\mathrm{CCH}_{3}\right), 25.5\left(\mathrm{CCH}_{3}\right), 44.2$ $\left(\mathrm{N}\left(\mathrm{CH}_{3}\right)_{2}\right), 44.6\left(\mathrm{NCH}_{2} \mathrm{CH}_{2}\right), 55.0\left(\mathrm{NCH}_{2} \mathrm{CH}_{2}\right), 98.1(\mathrm{CH}), 174.3(\mathrm{CN})$, 182.5 (CO). IR: v 2916, 1581, 1522, 1181, 1029, 941, 754, 658, 535, 440 $\mathrm{cm}^{-1}$.

L1 ${ }^{\text {Do2 }} \mathrm{AIMe}_{2}$ 8: Reagent: $3.17 \mathrm{~g}(16 \mathrm{mmoL}) \mathrm{L} 1{ }^{\mathrm{Do}} \mathrm{H}$. Yield $3.44 \mathrm{~g}(91 \%)$. $\mathrm{Mp} 30^{\circ} \mathrm{C}$. Elemental analysis calcd (\%) for $\mathrm{C}_{13} \mathrm{H}_{27} \mathrm{~N}_{2} \mathrm{OAl}$ : C 61.4, $\mathrm{H} 10.7$, $\mathrm{N}$ 11.0; found (\%): C 61.2, H 10.6, N 10.9. ${ }^{1} \mathrm{H}-\mathrm{NMR}$ (300 MHz, $\mathrm{C}_{6} \mathrm{D}_{6}$, $\left.25{ }^{\circ} \mathrm{C}\right): \delta=-0.27\left(\mathrm{~s}, 6 \mathrm{H}, \mathrm{Al}\left(\mathrm{CH}_{3}\right)_{2}\right), 0.85\left(\mathrm{t},{ }^{3} \mathrm{JHH}_{\mathrm{HH}}=7.3 \mathrm{~Hz}, 6 \mathrm{H}\right.$, $\left.\mathrm{N}\left(\mathrm{CH}_{2} \mathrm{CH}_{3}\right)_{2}\right), 1.45\left(\mathrm{~s}, 3 \mathrm{H}, \mathrm{CCH}_{3}\right), 1.78\left(\mathrm{~s}, 3 \mathrm{H}, \mathrm{CCH}_{3}\right), 2.31\left(\mathrm{q},{ }^{3} \mathrm{JHH}=7.0\right.$ $\left.\mathrm{Hz}, 4 \mathrm{H}, \mathrm{N}\left(\mathrm{CH}_{2} \mathrm{CH}_{3}\right)_{2}\right), 2.39\left(\mathrm{t},{ }^{3} \mathrm{~J}_{\mathrm{HH}}=7.8 \mathrm{~Hz}, 2 \mathrm{H},\left(\mathrm{NCH}_{2} \mathrm{CH}_{2}\right), 3.10\left(\mathrm{t},{ }^{3} \mathrm{JHH}_{\mathrm{HH}}\right.\right.$ $\left.=7.5 \mathrm{~Hz}, 2 \mathrm{H}, \mathrm{NCH}_{2} \mathrm{CH}_{2}\right), 4.73(\mathrm{~s}, 1 \mathrm{H}, \mathrm{CH}) .{ }^{13} \mathrm{C}-\mathrm{NMR}\left(75 \mathrm{MHz}, \mathrm{C}_{6} \mathrm{D}_{6}\right.$, $\left.25^{\circ} \mathrm{C}\right): \delta=-8.9\left(\mathrm{Al}\left(\mathrm{CH}_{3}\right)_{2}\right), 11.7\left(\mathrm{~N}\left(\mathrm{CH}_{2} \mathrm{CH}_{3}\right)_{2}\right), 20.7\left(\mathrm{CCH}_{3}\right), 25.4\left(\mathrm{CCH}_{3}\right)$

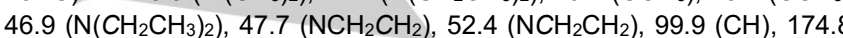

(CN), 179.7 (CO). IR: $v=2970,2926,2814,1602,1515,1404,1186,674$ $\mathrm{cm}^{-1}$.

L2 $^{\text {Do1 }}$ AlMe $_{2}$ 9: Reagent: $2.95 \mathrm{~g}$ (16 mmoL) L2 ${ }^{\mathrm{Do1}} \mathrm{H}$. Yield $3.70 \mathrm{~g}(95 \%)$. Mp. $26{ }^{\circ} \mathrm{C}$. Elemental analysis calcd (\%) for $\mathrm{C}_{12} \mathrm{H}_{25} \mathrm{~N}_{2} \mathrm{OAl}$ : C 60.0, $\mathrm{H} 10.5$, $\mathrm{N} 11.7$; found (\%): C 60.3, $\mathrm{H}$ 10.7, N 11.6. ${ }^{1} \mathrm{H}$ NMR $\left(300 \mathrm{MHz}, \mathrm{CD}_{2} \mathrm{Cl}_{2}\right.$, $\left.25^{\circ} \mathrm{C}\right): \delta=-0.85\left(\mathrm{~s}, 6 \mathrm{H}, \mathrm{Al}\left(\mathrm{CH}_{3}\right)_{2}\right), 1.71\left(\mathrm{~m}, 2 \mathrm{H}, \mathrm{NCH}_{2} \mathrm{CH}_{2}\right), 1.95(\mathrm{~s}, 3 \mathrm{H}$, $\left.\mathrm{CCH}_{3}\right), 2.08\left(\mathrm{~s}, 3 \mathrm{H}, \mathrm{CCH}_{3}\right), 2.21\left(\mathrm{~s}, 6 \mathrm{H}, \mathrm{N}\left(\mathrm{CH}_{3}\right)_{2}\right), 2.28\left(\mathrm{t},{ }^{3} \mathrm{JHH}_{\mathrm{HH}}=6.8 \mathrm{~Hz}\right.$ $2 \mathrm{H}, \mathrm{NCH}_{2} \mathrm{CH}_{2}$ ), $3.38\left(\mathrm{t},{ }^{3} \mathrm{JHH}_{\mathrm{HH}}=6.8 \mathrm{~Hz}, 2 \mathrm{H}, \mathrm{NCH}_{2} \mathrm{CH}_{2}\right), 5.07(\mathrm{~s}, 1 \mathrm{H}, \mathrm{CH})$ ${ }^{13} \mathrm{C}$ NMR $\left(75 \mathrm{MHz}, \mathrm{CD}_{2} \mathrm{Cl}_{2}, 25{ }^{\circ} \mathrm{C}\right): \delta=-10.5\left(\mathrm{Al}\left(\mathrm{CH}_{3}\right)_{2}\right), 20.6\left(\mathrm{CCH}_{3}\right)$

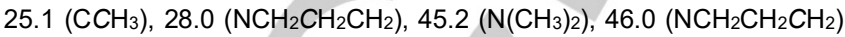
$56.9\left(\mathrm{NCH}_{2} \mathrm{CH}_{2} \mathrm{CH}_{2}\right), 99.8(\mathrm{CH}), 174.6(\mathrm{CN}), 177.7$ (CO). ATR-IR: v 2928, 2886, 2859, 2816, 2767, 2723, 1600, 1515, 1458, 1405, 1186, 946, $769,713,674,580,387 \mathrm{~cm}^{-1}$

Synthesis of heteroleptic complexes $\mathrm{L}^{\mathrm{Do}}{ }^{\mathrm{M}} \mathrm{M}\left(\mathrm{Me}_{2}\right) \mathrm{Cp}^{*}(\mathrm{M}=\mathrm{Ti}, \mathrm{Zr})$ : A solution of $\mathrm{L} 1{ }^{\mathrm{Do}} \mathrm{H}(0.65 \mathrm{~g}, 3.3 \mathrm{mmol})$ in $5 \mathrm{~mL}$ of $n$-pentane was slowly added to a cooled $\left(-60{ }^{\circ} \mathrm{C}\right)$ solution of $\mathrm{Cp}^{*} \mathrm{MMe}_{3}(0.75 \mathrm{~g}, 3.3 \mathrm{mmol})$ in 10 $\mathrm{mL}$ of $n$-pentane. The resulting red solutions were stirred for $2 \mathrm{~h}$ at $-60^{\circ} \mathrm{C}$ and then warmed to ambient temperature. The solvent was reduced in vacuo to $3 \mathrm{~mL}$ and the solution was stored at $-30{ }^{\circ} \mathrm{C}$, yielding red crystalline solids after $24 \mathrm{~h}$.

L1 ${ }^{\text {Do2}} \mathbf{T i}\left(\mathrm{Me}_{2}\right) \mathbf{C p}^{*}$ 10: Reagent: $0.75 \mathrm{~g}$ (3.3 mmol) $\mathrm{Cp}^{*} \mathrm{TiMe}_{3}$. Yield $1.02 \mathrm{~g}$ (95\%). Mp $54{ }^{\circ} \mathrm{C}$. Elemental analysis calcd (\%) for $\mathrm{C}_{23} \mathrm{H}_{42} \mathrm{~N}_{2} \mathrm{OTi}$ : C 65.7 , H 10.0, N 7.3; found (\%): C 65.6, H 10.2, N 7.1. ${ }^{1} \mathrm{H}$ NMR $\left(300 \mathrm{MHz}, \mathrm{C}_{6} \mathrm{D}_{6}\right.$, $\left.25^{\circ} \mathrm{C}\right): \delta=0.83\left(\mathrm{~s}, 6 \mathrm{H}, \mathrm{Ti}\left(\mathrm{CH}_{3}\right)_{2}\right), 0.92\left(\mathrm{t},{ }^{3} \mathrm{~J}_{\mathrm{HH}}=7.1 \mathrm{~Hz}, 6 \mathrm{H}, \mathrm{N}\left(\mathrm{CH}_{2} \mathrm{CH}_{3}\right)_{2}\right)$, $1.88\left(\mathrm{~s}, 15 \mathrm{H}, \mathrm{C}_{5}\left(\mathrm{CH}_{3}\right)_{5}\right), 1.91\left(\mathrm{~s}, 3 \mathrm{H}, \mathrm{CCH}_{3}\right), 1.98\left(\mathrm{~s}, 3 \mathrm{H}, \mathrm{CCH}_{3}\right), 2.35$ (q, $\left.4 \mathrm{H}, \mathrm{N}\left(\mathrm{CH}_{2} \mathrm{CH}_{3}\right)_{2}\right), 2.38\left(\mathrm{~m}, 2 \mathrm{H}, \mathrm{NCH}_{2} \mathrm{CH}_{2}\right), 3.26\left(\mathrm{~m}, 2 \mathrm{H}, \mathrm{NCH}_{2} \mathrm{CH}_{2}\right), 5.23$ (s, $1 \mathrm{H}, \mathrm{CH}) .{ }^{13} \mathrm{C}$ NMR $\left(75 \mathrm{MHz}, \mathrm{C}_{6} \mathrm{D}_{6}, 25{ }^{\circ} \mathrm{C}\right): \delta=12.4\left(\mathrm{C}_{5}\left(\mathrm{CH}_{3}\right) 5\right), 12.8$ $\left(\mathrm{N}\left(\mathrm{CH}_{2} \mathrm{CH}_{3}\right)_{2}\right), 23.3\left(\mathrm{CCH}_{3}\right), 25.8\left(\mathrm{CCH}_{3}\right), \quad 48.1 \quad\left(\mathrm{NCH}_{2} \mathrm{CH}_{2}\right), \quad 53.1$

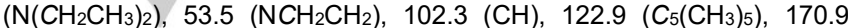
(CN), 176.7 (CO). IR: v 2969, 2801, 1584, 1508, 1370, 1281, 1201, 1068, $1000,952,791,675,483,432$.

L1 ${ }^{\text {Do22Zr(Me }}$ )Cp* 11: Reagent: $0.90 \mathrm{~g}(3.3 \mathrm{mmol}) \mathrm{Cp}^{*} \mathrm{ZrMe}$. Yield $0.31 \mathrm{~g}$ (61\%). Mp $62{ }^{\circ} \mathrm{C}$. Elemental analysis calcd (\%) for $\mathrm{C}_{23} \mathrm{H}_{42} \mathrm{~N}_{2} \mathrm{OZr}$ : C 60.8 , H 9.3, N 6.2; found (\%): C 60.7, H 9.3, N 6.0. ${ }^{1} \mathrm{H}$ NMR (300 MHz, $\mathrm{C}_{6} \mathrm{D}_{6}$, $\left.25{ }^{\circ} \mathrm{C}\right): \delta=0.44\left(\mathrm{~s}, 6 \mathrm{H}, \quad \mathrm{Zr}\left(\mathrm{CH}_{3}\right)_{2}\right), 0.93\left(\mathrm{t},{ }^{3} \mathrm{JHH}_{\mathrm{HH}}=7.1 \mathrm{~Hz}, 6 \mathrm{H}\right.$ $\left.\mathrm{N}\left(\mathrm{CH}_{2} \mathrm{CH}_{3}\right)_{2}\right), 1.78\left(\mathrm{~s}, 3 \mathrm{H}, \mathrm{CCH}_{3}\right), 1.85\left(\mathrm{~s}, 3 \mathrm{H}, \mathrm{CCH}_{3}\right), 1.92(\mathrm{~s}, 15 \mathrm{H}$, $\left.\mathrm{C}_{5}\left(\mathrm{CH}_{3}\right)_{5}\right), 2.37\left(\mathrm{q},{ }^{3} \mathrm{JHH}_{\mathrm{HH}}=7.1 \mathrm{~Hz}, 2 \mathrm{H} \mathrm{N}\left(\mathrm{CH}_{2} \mathrm{CH}_{3}\right)_{2}\right), 2.40(\mathrm{~m}, 4 \mathrm{H}$ $\left.\mathrm{NCH}_{2} \mathrm{CH}_{2}\right), 2.40\left(\mathrm{~m}, 2 \mathrm{H}, \mathrm{NCH}_{2} \mathrm{CH}_{2}\right), 3.44\left(\mathrm{~m}, 2 \mathrm{H}, \mathrm{NCH}_{2} \mathrm{CH}_{2}\right), 5.03(\mathrm{~s}, 1 \mathrm{H}$ $\mathrm{CH}) .{ }^{13} \mathrm{C}$ NMR $\left(75 \mathrm{MHz}, \mathrm{C}_{6} \mathrm{D}_{6}, 25{ }^{\circ} \mathrm{C}\right): \delta=11.0\left(\mathrm{C}_{5}\left(\mathrm{CH}_{3}\right)_{5}\right), 12.1$ $\left(\mathrm{N}\left(\mathrm{CH}_{2} \mathrm{CH}_{3}\right)_{2}\right), \quad 22.2\left(\mathrm{CCH}_{3}\right), \quad 25.0\left(\mathrm{CCH}_{3}\right), \quad 47.3\left(\mathrm{NCH}_{2} \mathrm{CH}_{2}\right), \quad 51.0$ $\left(\mathrm{N}\left(\mathrm{CH}_{2} \mathrm{CH}_{3}\right)_{2}\right), 53.0\left(\mathrm{NCH}_{2} \mathrm{CH}_{2}\right), 102.3(\mathrm{CH}), 119.0\left(\mathrm{C}_{5}\left(\mathrm{CH}_{3}\right)_{5}\right), 172.1$ (CN), 174.9 (CO). IR: v 2912, 1584, 1509, 1396, 1281, 1067, 1000, 948, $799,675,552,464,420$.

Synthesis of $\mathrm{L}^{\mathrm{Do} 1} \mathrm{Al}(\mathrm{Cl}) \mathrm{Me}$ 12: A solution of ethereal $\mathrm{HCl}(1 \mathrm{~mL}, 2 \mathrm{~N}, 2$ $\mathrm{mmol}$ ) was slowly added to a solution of $0.45 \mathrm{~g}(2.0 \mathrm{mmol}) \mathrm{L}^{\mathrm{Do}} \mathrm{AlMe}_{2} 7$ in $15 \mathrm{~mL}$ of $\mathrm{CH}_{2} \mathrm{Cl}_{2}$. The solution was stirred for $2 \mathrm{~h}$, until gas evolution has stopped. The solvent was removed in vacuo and the resulting lightyellow solid was recrystallized from a solution in toluene. Light-yellow crystals of 12 were formed within $24 \mathrm{~h}$ upon storage at $-30{ }^{\circ} \mathrm{C}$. Yield 0.43 g $(87 \%)$. Mp $117^{\circ} \mathrm{C}$. Elemental analysis calcd (\%) for $\mathrm{C}_{10} \mathrm{H}_{20} \mathrm{AlClN}_{2} \mathrm{O}: \mathrm{C}$ 48.7, $\mathrm{H}$ 8.2, N 11.4; found (\%): C 49.1, H 8.1, N 11.2. ${ }^{1} \mathrm{H}$ NMR (300 MHz, $\left.\mathrm{CD}_{2} \mathrm{Cl}_{2}, 25^{\circ} \mathrm{C}\right): \delta=-0.81\left(\mathrm{~s}, 3 \mathrm{H}, \mathrm{Al}\left(\mathrm{CH}_{3}\right)_{2}\right), 1.94\left(\mathrm{~s}, 3 \mathrm{H}, \mathrm{CCH}_{3}\right), 2.03(\mathrm{~s}$ $\left.3 \mathrm{H}, \mathrm{CCH}_{3}\right), 2.36\left(\mathrm{~s}, 6 \mathrm{H}, \mathrm{N}\left(\mathrm{CH}_{3}\right)_{2}\right), 2.84\left(\mathrm{t},{ }^{3}{ }_{\mathrm{HH}}=6.4 \mathrm{~Hz}, 2 \mathrm{H}, \mathrm{NCH}_{2} \mathrm{CH}_{2}\right)$, $3.37\left(\mathrm{t},{ }^{3} \mathrm{JHH}_{\mathrm{HH}}=6.4 \mathrm{~Hz}, 2 \mathrm{H}, \mathrm{NCH}_{2} \mathrm{CH}_{2}\right), 5.17(\mathrm{~s}, 1 \mathrm{H}, \mathrm{CH}) \cdot{ }^{13} \mathrm{C} \mathrm{NMR}(75$ $\left.\mathrm{MHz}, \mathrm{CD}_{2} \mathrm{Cl}_{2}, 25^{\circ} \mathrm{C}\right): \delta=22.9\left(\mathrm{CCH}_{3}\right), 25.4\left(\mathrm{CCH}_{3}\right), 44.1\left(\mathrm{~N}\left(\mathrm{CH}_{3}\right)_{2}\right), 44.9$ $\left(\mathrm{NCH}_{2} \mathrm{CH}_{2}\right), 54.5\left(\mathrm{NCH}_{2} \mathrm{CH}_{2}\right), 99.0(\mathrm{CH}), 177.0(\mathrm{CN}), 182.4(\mathrm{CO}) . \mathrm{IR}: \mathrm{v}$ $1586,1526,1433,1396,1344,1300,1181,1036,944,770,679,652$ $611,464$. 
$\mathrm{L1}^{\mathrm{Do} 1} \mathrm{AICl}_{2}$ 13: A solution of $\mathrm{GaCl}_{3}(0.13 \mathrm{~g}, 0.75 \mathrm{mmol})$ in $5 \mathrm{~mL}$ of $\mathrm{CH}_{2} \mathrm{Cl}_{2}$ was added to a solution of $0.18 \mathrm{~g}(0.76 \mathrm{mmol}) 12$ in $5 \mathrm{ml} \mathrm{of} \mathrm{CH}_{2} \mathrm{Cl}_{2}$ at room temperature and the resulting solution was stirred for additional 30 minutes. Thereafter, all volatiles were removed in vacuum yielding a red solid. Colorless crystals were formed after storage of a solution in $\mathrm{CH}_{2} \mathrm{Cl}_{2}$ at $0{ }^{\circ} \mathrm{C}$ for $12 \mathrm{~h}$. Yield $0.152 \mathrm{~g}(75 \%)$. Mp $172{ }^{\circ} \mathrm{C}$. Elemental analysis calcd (\%) for $\mathrm{C}_{9} \mathrm{H}_{17} \mathrm{AIN}_{2} \mathrm{O}$ : C 40.5, H 6.1, N 10.1; found (\%): C 39.8, H 6.3, $\mathrm{N}$ 9.6. ${ }^{1} \mathrm{H}\left(300 \mathrm{MHz}, \mathrm{CDCl}_{3}, 25{ }^{\circ} \mathrm{C}\right): \delta=2.02\left(\mathrm{~s}, 3 \mathrm{H}, \mathrm{CCH}_{3}\right), 2.05(\mathrm{~s}, 3 \mathrm{H}$, $\left.\mathrm{CCH}_{3}\right), 2.54\left(\mathrm{~s}, 6 \mathrm{H}, \mathrm{N}\left(\mathrm{CH}_{3}\right)_{2}\right), 3.01\left(\mathrm{t},{ }^{3} \mathrm{JHH}_{\mathrm{HH}}=6.5 \mathrm{~Hz}, 2 \mathrm{H}, \mathrm{CH}_{2} \mathrm{~N}(\mathrm{CH})_{2}\right)$, $3.44\left(\mathrm{t},{ }^{3} \mathrm{~J}_{\mathrm{HH}}=6.4 \mathrm{~Hz}, 2 \mathrm{H}, \mathrm{CNCH}_{2}\right), 5.25(\mathrm{~s}, 1 \mathrm{H}, \mathrm{CH}) \cdot{ }^{13} \mathrm{C}\left(75 \mathrm{MHz} \mathrm{CDCl}_{3}\right.$, $\left.25^{\circ} \mathrm{C}\right): \delta=23.4\left(\mathrm{CCH}_{3}\right), 26.0\left(\mathrm{CCH}_{3}\right), 43.7\left(\mathrm{CH}_{2} \mathrm{~N}\left(\mathrm{CH}_{3}\right)_{2}\right), 46.0\left(\mathrm{~N}\left(\mathrm{CH}_{3}\right)_{2}\right)$ $100.7(\mathrm{CH}), 177.9(\mathrm{CN}), 183.9(\mathrm{CO}) . \mathrm{IR}: \mathrm{v} 1590,1528,1397,1346,1306$, $1017,957,768,683,492,456,417$

Synthesis of $\mathrm{L} 1^{\mathrm{Do} 1} \mathrm{Al}(\mathrm{Me}) \mathrm{O}-2,6-\mathrm{Me}_{2} \mathrm{C}_{6} \mathrm{H}_{3}$ 14: A solution of 2,6dimethyphenol $(0.24 \mathrm{~g}(2 \mathrm{mmol})$ in $4 \mathrm{~mL}$ of toluene was added to a solution of $0.45 \mathrm{~g}(2.0 \mathrm{mmol}) \mathrm{L}^{\mathrm{Do}^{\mathrm{D}} \mathrm{AlMe}_{2}} 7 \mathrm{in} 10 \mathrm{~mL}$ of toluene. The solution was warmed to ambient temperature and stirred for one additional hour. The solvent was reduced in vacuo to $2 \mathrm{~mL}$ and the solution stored at $-10^{\circ} \mathrm{C}$, yielding colorless crystalline solids. Yield $0.51 \mathrm{~g}$ (77\%). Mp $97^{\circ} \mathrm{C}$. Elemental analysis calcd (\%) for $\mathrm{C}_{18} \mathrm{H}_{19} \mathrm{AlN}_{2} \mathrm{O}_{2}$ : C 65.0 , $\mathrm{H}$ 8.8, N 8.4; found (\%): C 64.7, $\mathrm{H}$ 8.7, N 8.4. ${ }^{1} \mathrm{H}$ NMR (300 MHz, $\mathrm{CD}_{2} \mathrm{Cl}_{2}$, $\left.25^{\circ} \mathrm{C}\right): \delta=-0.84\left(\mathrm{~s}, 3 \mathrm{H}, \mathrm{AlCH}_{3}\right), 1.52\left(\mathrm{~s}, 3 \mathrm{H}, \mathrm{CCH}_{3}\right), 2.02\left(\mathrm{~s}, 3 \mathrm{H}, \mathrm{CCH}_{3}\right)$, $2.06\left(\mathrm{~s}, 6 \mathrm{H}, \mathrm{C}_{\mathrm{Ar}} \mathrm{CH}_{3}\right), 2.46\left(\mathrm{~s}, 6 \mathrm{H}, \mathrm{N}\left(\mathrm{CH}_{3}\right)_{2}\right), 2.90\left(\mathrm{~m}, 2 \mathrm{H}, \mathrm{NCH}_{2} \mathrm{CH}_{2}\right), 3.44$ $\left(\mathrm{m}, 2 \mathrm{H}, \mathrm{NCH}_{2} \mathrm{CH}_{2}\right), 5.05(\mathrm{~s}, 1 \mathrm{H}, \mathrm{CH}), 6.45\left(\mathrm{t},{ }^{3} \mathrm{~J}_{\mathrm{HH}}=7.4 \mathrm{~Hz}, 1 \mathrm{H}, \mathrm{CH}\right), 6.81$ (d, $\left.{ }^{3} \mathrm{~J}_{\mathrm{HH}}=7.4 \mathrm{~Hz}, 2 \mathrm{H}, m-\mathrm{CH}\right) \cdot{ }^{13} \mathrm{C} \mathrm{NMR}\left(75 \mathrm{MHz}, \mathrm{CD}_{2} \mathrm{Cl}_{2}, 25^{\circ} \mathrm{C}\right): \delta=$ $17.9\left(\mathrm{C}_{\mathrm{Ar}} \mathrm{CH}_{3}\right), \quad 23.1\left(\mathrm{CCH}_{3}\right), \quad 25.4\left(\mathrm{CCH}_{3}\right), \quad 45.0 \quad\left(\mathrm{~N}\left(\mathrm{CH}_{3}\right)_{2}\right), \quad 45.8$ $\left(\mathrm{NCH}_{2} \mathrm{CH}_{2}\right), 55.3\left(\mathrm{NCH}_{2} \mathrm{CH}_{2}\right), 98.9(\mathrm{CH}), 116.6\left(C_{A r-p} \mathrm{H}\right), 127.8\left(C_{A r-q} \mathrm{CH}_{3}\right)$, $128.0\left(C_{m} \mathrm{H}\right), 159.2\left(C_{A r} \mathrm{O}\right), 176.4(\mathrm{CN}), 182.9(\mathrm{CO}) . \mathrm{IR}: \mathrm{v} 2914,1580$, 1522, 1461, 1397, 1341, 1278, 1237, 1189, 1091, 1027, 954, 868, 791, $751,719,654,621,584,543,470$.

Synthesis of $\quad \mathrm{L}^{\mathrm{Do}}{ }^{\mathrm{Al}} \mathrm{Al}\left(\mathrm{O}-2,6-\mathrm{Me}_{2} \mathrm{C}_{6} \mathrm{H}_{3}\right)_{2} \quad$ 15: A solution of 2,6dimethyphenol $(0.49 \mathrm{~g}(4 \mathrm{mmol})$ in $4 \mathrm{~mL}$ of toluene was added to a solution of $0.45 \mathrm{~g}(2.0 \mathrm{mmol}) \mathrm{L}^{\mathrm{Do}^{\mathrm{D}} \mathrm{AlMe}_{2}} 7 \mathrm{in} 10 \mathrm{~mL}$ of toluene. The solution was warmed to ambient temperature and stirred for one additional hour. The solvent was reduced in vacuo to $2 \mathrm{~mL}$ and the solution stored at $-10^{\circ} \mathrm{C}$, yielding colorless crystalline solids. Yield $0.79 \mathrm{~g}$ (90\%). Mp. $197^{\circ} \mathrm{C}$. Elemental analysis calcd (\%) for $\mathrm{C}_{25} \mathrm{H}_{35} \mathrm{AIN}_{2} \mathrm{O}_{3}$ : C 68.5, H 8.0, N 6.4; found (\%): C 68.8, H 8.1, N 6.4. ${ }^{1} \mathrm{H}$ NMR $(300 \mathrm{MHz}$, $\left.\mathrm{CD}_{2} \mathrm{Cl}_{2}, 25^{\circ} \mathrm{C}\right): \delta=1.27\left(\mathrm{~s}, 3 \mathrm{H}, \mathrm{CH}_{3}\right), 2.07\left(\mathrm{~s}, 3 \mathrm{H}, \mathrm{CH}_{3}\right), 2.11(\mathrm{~s}, 12 \mathrm{H}$, $\left.\mathrm{C}_{\mathrm{Ar}} \mathrm{CH}_{3}\right), 2.70\left(\mathrm{~s}, 6 \mathrm{H}, \mathrm{N}\left(\mathrm{CH}_{3}\right)_{2}\right), 3.00\left(\mathrm{t},{ }^{3} \mathrm{~J}_{\mathrm{HH}}=6.5 \mathrm{~Hz}, 2 \mathrm{H}, \mathrm{NCH}_{2} \mathrm{CH}_{2}\right.$ ), $3.56\left(\mathrm{t},{ }^{3} \mathrm{~J}_{\mathrm{HH}}=6.5 \mathrm{~Hz}, 2 \mathrm{H}, \mathrm{NCH}_{2} \mathrm{CH}_{2}\right), 5.09(\mathrm{~s}, 1 \mathrm{H}, \mathrm{CH}), 6.49\left(\mathrm{t},{ }^{3} \mathrm{~J}_{\mathrm{HH}}=7.3\right.$ $\mathrm{Hz}, 1 \mathrm{H}, p-\mathrm{CH}), 6.82\left(\mathrm{~d},{ }^{3} \mathrm{~J}_{\mathrm{HH}}=7.3 \mathrm{~Hz}, 2 \mathrm{H}, m-\mathrm{CH}\right) \cdot{ }^{13} \mathrm{C} \mathrm{NMR}(75 \mathrm{MHz}$, $\left.\mathrm{CD}_{2} \mathrm{Cl}_{2}, 25{ }^{\circ} \mathrm{C}\right): \delta=18.1\left(\mathrm{C}_{\mathrm{ArCH}}\right), 23.1\left(\mathrm{CCH}_{3}\right), 24.9\left(\mathrm{CCH}_{3}\right), 44.7$ $\left(\mathrm{N}\left(\mathrm{CH}_{3}\right)_{2}\right), 47.1\left(\mathrm{NCH}_{2} \mathrm{CH}_{2}\right), 56.0\left(\mathrm{NCH}_{2} \mathrm{CH}_{2}\right), 99.7(\mathrm{CH}), 117.2\left(C_{A r-p} \mathrm{H}\right)$, $127.7\left(\mathrm{C}_{\mathrm{Ar}} \mathrm{CH}_{3}\right), 128.4\left(\mathrm{C}_{m} \mathrm{H}\right), 158.2\left(\mathrm{C}_{\mathrm{Ar}} \mathrm{O}\right), 176.9(\mathrm{CN}), 183.8(\mathrm{CO}) . \mathrm{IR}: \mathrm{v}$ $1583,1460,1393,1339,1275,1237,1092,1028,953,902,876,789$, $751,736,681,615,575,487$.

Single crystal X-ray diffraction. Crystallographic data of 2, 4, 7, 10 and 12-14, which were collected on a Bruker AXS D8 Kappa diffractometer with APEX2 detector (MoK $\alpha$ radiation, $\lambda=0.71073 \AA$ ) at $100(2) \mathrm{K}$, are summarized in Table S1 (SI). The solid-state structures of 2, 4, 7, 10 and 12-14 are shown in Figures 1 to 7, bond lengths and angles of 2, 4, 7, 10 and 12-14 are summarized in Tables 1 and 2. The structures were solved by Direct Methods (SHELXS-97) and refined anisotropically by full-matrix least-squares on $F^{2}$ (SHELXL-2014). ${ }^{[26,27]}$ Absorption corrections were performed semi-empirically from equivalent reflections on basis of multiscans (Bruker AXS APEX2).

Hydrogen atoms were refined using a riding model or rigid methyl groups. In 12 the toluene molecule is disordered over a center of inversion. Its atoms' adp were restraint with RIGU. The methyl and chlorine substituent are disordered. The carbon atom could only be refined isotropically and the geometric parameters of the disordered parts cannot be regarded meaningful. Lowering the symmetry does not resolve the disorder.

The crystallographic data of 2, 4, 7, 10 and 12-14 (excluding structure factors) have been deposited with the Cambridge Crystallographic Data Centre as supplementary publication nos. CCDC-1855741 (2), -1855740 (4), -1855739 (7), -1855742 (10), -1855743 (12), -1855744 (13) and 1855745 (14).Copies of the data can be obtained free of charge on application to CCDC, 12 Union Road, Cambridge, CB21EZ (fax: (+44) 1223/336033; e-mail: deposit@ccdc.cam-ak.uk).

Supporting Information (see footnote on the first page of this article): A CIF file giving X-ray crystallographic data of 2, 4, 7, 10, 12-14, spectroscopic details $\left({ }^{1} \mathrm{H},{ }^{13} \mathrm{C}\right.$ NMR; IR) and crystallographic details of 2 , $4,7,10,12-14$

\section{Acknowledgements}

Stephan Schulz gratefully acknowledges financial support by the University of Duisburg-Essen and Evonik Industry.

Keywords: Zinc $•$ aluminum $\bullet$ titanium $\bullet \beta$-ketoiminate

[1] a) S. Kumar, D.N. Dhar, P.N. Saxena, J. Sci. Ind. Res. 2009, 68, 181187; b) A. Blagus, D. Cinčić, T. Friščić, B. Kaitner, V. Stilinović, Macedonian J. Chem. Chem. Eng. 2010, 29, 117-138; c) C. M. da Silva, D. L. da Silva, L. V. Modolo, R. B. Alves, M. A. de Resende, C. V.B. Martins, Â. de Fátima J. Adv. Res. 2011, 2, 1-8; d) B. Govindh, B. S Diwakar, Y. L N Murthy, Org. Commun. 2012, 5, 105-119; e) W. Qin, S. Long, M. Panunzio, S. Biondi, Molecules 2013, 18, 12264-12289; f) C J. Dhanaraj, J. Johnson, J. Joseph, R. S. Joseyphus, J. Coord. Chem. 2013, 66, 1416-1450; g) K. A. Maher, S. R. Mohammed, IJCRR 2015, 7, 06-16; h) S. Akhter, H. UI Zaman, S. Mir, A. Mahmood Dar, S. Shrivastava, Eur. Chem. Bull. 2017, 6, 475-483; i) Pa. Gull, A. A Hashmi, Asian J. Phys. Chem. Sci. 2017, 2, 2456-7779

[2] a) B. J. O'Keefe, M. A. Hillmyer, W. B. Tolman, Dalton Trans. 2001, 2215-2224; b) O. Dechy-Cabaret, B. Martin-Vaca, D. Bourissou, Chem. Rev. 2004, 104, 6147-6176; c) J. Wu, T.-L. Yu, C. T. Chen, C.-C. Lin, Coord. Chem. Rev. 2006, 250, 602-626; d) R. H. Platel, L. M. Hodgson C. K. Williams, Polym. Rev. 2008, 48, 11-63; e) M. J. Stanford, A. P Dove, Chem. Soc. Rev. 2010, 39, 486-494.

[3] a) H. Y. Chen, H. Y. Tang, C. C. Lin, Macromolecules 2006, 39, 37453752; b) V. Poirier, T. Roisnel, J.-F. Carpentier, Y. Sarazin, Dalton Trans. 2011, 40, 523-534; c) R. H. Platel, A. J. P. White, C. K. Williams, Inorg. Chem. 2011, 50, 7718-7728; d) M. Bouyahyi, N. Ajellal, E. Kirillov, C. M. Thomas, J.-F. Carpentier, Chem. Eur. J. 2011, 17, 1872-1883; e) T.-P.-A. Cao, A. Buchard, X. F. Le Goff, A. Auffrant, C. K. Williams, Inorg. Chem. 2012, 51, 2157-2169; f) S. Song, X. Zhang, H. Ma, Y. Yang, Dalton Trans. 2012, 41, 3266-3277; g) Y. Wang, H. Ma, Chem. Commun. 2012, 48, 6729-6731; h) W. Zhao, Y. Wang, X. Liu, X. Chen, D. Cui, E. Y.-X. Chen, Chem. Commun. 2012, 48, 6375-6377; i) H. Wang, H. Ma, Chem. Commun. 2013, 49, 8686-8688; j) R. Jianming, X. Anguo, W. Hongwei, Y. Hailin, Designed Monomers and Polymers 2014, 17, 345-355; k) D. Jędrzkiewicz, J. Ejfler, N. Gulia, Ł. John, S. Szafert, Dalton Trans. 2015, 44, 13700-13715; I) D. Jędrzkiewicz, J. Ejfler, N. Gulia, Ł. John, S. Szafert, Dalton Trans. 2016, 45, 2829-2838.

[4] C. G. Gianopoulos, N. Kumar, Y. Zhao, L. Jia, K. Kirschbaum, M. R. Mason, Dalton Trans. 2016, 45, 13787-13797.

[5] M. Cheng, A. B. Attygalle, E. B. Lobkovsky, G. W. Coates, J. Am Chem. Soc. 1999, 121, 11583-11584. 
[6] a) Maya, A. Maity, T. S. Teets, Organometallics 2016, 35, 2890-2899; b) D. S. Laitar, C. J. N. Mathison, W. M. Davis, J. P. Sadighi, Inorg Chem. 2003, 42, 7354-7356; c) D. C. H. Do, A. Keyser, A. V. Protchenko, B. Maitland, I. Pernik, H. Niu, E. L. Kolychev, A. Rit, D Vidovic, A. Stasch, C. Jones, S. Aldridge, Chem. Eur. J. 2017, 23 5830-5841; d) K. Huse, C. Wölper, S. Schulz, Eur. J. Inorg. Chem 2018, in the press, DOI: $10.1002 /$ ejic. 201800414.

[7] a) L. Czympiel, J. Pfrommer, W. Tyrra, M. Schäfer, S. Mathur, Inorg. Chem. 2015, 54, 25-37; b) C. Di lulio, M. Middleton, G. Kociok-Köhn, M. D. Jones, A. L. Johnson, Eur. J. Inorg. Chem. 2013, 1541-1554; c) J. Lewiński, P. Goś, T. Kopeć, J. Lipkowski, R. Luboradzki, Inorg Chem. Commun. 1999, 2, 374-377; d) J. Lewiński, S. Pasynkiewicz, J. Lipkowski, Inorg. Chim. Acta 1990, 178, 113-123; e) J. H. Wengrovius, M. F. Garbauskas, E. A. Williams, R. C. Going, P. E. Donahue, J. F. Smith, J. Am. Chem. Soc. 1986, 108, 982-989.

[8] C. Scheiper, D. Dittrich, C. Wölper, D. Bläser, J. Roll, S. Schulz, Eur. J. Inorg. Chem. 2014, 2230-2240.

[9] L. A. Lesikar, A. F. Gushwa, A. F. Richards, J. Organomet. Chem. 2008, 693, 3245-3255

[10] a) D. Pugh, P. Marchand, I. P. Parkin, C. J. Carmalt, Inorg. Chem. 2012, 51, 6385-6395; b) D. Neculai, H. W. Roesky, A. M. Neculai, J. Magull, H. G. Schmidt, M. Noltemeyer, J. Organomet. Chem. 2002, 643-644, 47-52

[11] 2: $\left[\mathrm{C}_{17} \mathrm{H}_{39} \mathrm{~N}_{3} \mathrm{OSi} \mathrm{Zn}_{2}\right], M=423.06$, colorless crystal, $(0.250 \times 0.134 \times$ $0.102 \mathrm{~mm})$; monoclinic, space group $P 2_{1} / c ; a=16.8067(4) \AA, b=$ 18.0457(4) $\AA, c=17.1775(4) \AA ; \alpha=90^{\circ}, \beta=117.4450(10)^{\circ}, \gamma=90^{\circ}, V$ $=4623.40(19) \AA^{3} ; Z=8 ; \mu=1.175 \mathrm{~mm}^{-1} ; \rho_{\text {calc }}=1.216 \mathrm{~g} \cdot \mathrm{cm}^{-3} ; 48147$ reflexes $\left(\theta_{\max }=30.577^{\circ}\right), 14035$ unique $\left(R_{\text {int }}=0.0324\right) ; 453$ parameters; largest max./min in the final difference Fourier synthesis $0.599 \mathrm{e} \cdot \AA^{-3 /} /-0.556 \mathrm{e} \cdot \AA^{-3} ;$ max./min. transmission $0.63 / 0.75 ; R_{1}=0.0343$ $(\mathrm{I}>2 \sigma(\mathrm{I})), w R_{2}=0.0861$ (all data). 4: $\left[\mathrm{C}_{14} \mathrm{H}_{26} \mathrm{~N}_{2} \mathrm{OZn}\right], M=303.74$ colorless crystal, $(0.390 \times 0.340 \times 0.280 \mathrm{~mm})$; monoclinic, space group $P 2{ }_{1} / c ; a=11.0419(12) \AA, b=11.2385(12) \AA, c=13.0253(13) \AA ; \alpha=$ $90^{\circ}, \beta=113.447(3)^{\circ}, \gamma=90^{\circ}, V=1482.9(3) \AA^{3} ; Z=4 ; \mu=1.648 \mathrm{~mm}^{-1}$; $\rho_{\text {calc }}=1.360 \mathrm{~g} \cdot \mathrm{cm}^{-3} ; 33130$ reflexes $\left(\theta_{\max }=29.658^{\circ}\right), 4114$ unique $\left(R_{\text {int }}=\right.$ $0.0316) ; 166$ parameters; largest max./min in the final difference Fourier synthesis 0.594 e. $\AA^{-3} / \quad-0.232$ e. $\AA^{-3} ;$ max./min. transmission $0.58 / 0.75 ; \quad R_{1}=0.0229(\mathrm{I}>2 \sigma(\mathrm{I})), \quad w R_{2}=0.0617$ (all data). 7: $\left[\mathrm{C}_{16} \mathrm{H}_{28} \mathrm{~N}_{2} \mathrm{O}_{4} \mathrm{Zn}\right], M=377.77$, colorless crystal, $(0.552 \times 0.328 \times 0.194$ $\mathrm{mm}$ ); orthorhombic, space group Pca2 $1 ; a=21.752(3) \AA, b=5.6648(7)$ $\AA, c=14.5039(19) \AA ; \alpha=90^{\circ}, \beta=90^{\circ}, \gamma=90^{\circ}, V=1787.2(4) \AA^{3} ; Z=4$; $\mu=1.395 \mathrm{~mm}^{-1} ; \rho_{\text {calc }}=1.404 \mathrm{~g} \cdot \mathrm{cm}^{-3} ; 37941$ reflexes $\left(\theta_{\max }=30.548^{\circ}\right)$, 5416 unique $\left(R_{\text {int }}=0.0406\right) ; 215$ parameters; Flack-Parameter $x=$ $0.197(12)$; largest max./min in the final difference Fourier synthesis $0.306 \mathrm{e} \cdot \AA^{-3} /-0.405 \mathrm{e} \cdot \AA^{-3} ;$ max./min. transmission $0.55 / 0.90 ; R_{1}=0.0258$ $(\mathrm{I}>2 \sigma(\mathrm{I})), w R_{2}=0.0591$ (all data). 8: $\left[\mathrm{C}_{11} \mathrm{H}_{23} \mathrm{AIN}_{2} \mathrm{O}\right], M=226.29$, orange crystal, $(0.430 \times 0.380 \times 0.320 \mathrm{~mm})$; monoclinic, space group $P 2_{1} / \mathrm{n}$; a $=8.1748(8) \AA, b=16.1174(15) \AA, c=10.4904(10) \AA ; \alpha=90^{\circ}, \beta=$ $97.528(2)^{\circ}, y=90^{\circ}, V=1370.3(2) \AA^{3} ; Z=4 ; \mu=0.129 \mathrm{~mm}^{-1} ; \rho_{\text {calc }}=$ $1.097 \mathrm{~g} \cdot \mathrm{cm}^{-3} ; 29264$ reflexes $\left(\theta_{\max }=25.242^{\circ}\right), 4766$ unique $\left(R_{\mathrm{int}}=\right.$ $0.0250) ; 136$ parameters; largest $\max . / \mathrm{min}$ in the final difference Fourier synthesis 0.459 e. $\AA^{-3} /-0.253$ e. $\AA^{-3} ;$ max./min. transmission $0.67 / 0.75 ; R_{1}=0.0338(\mathrm{I}>2 \sigma(\mathrm{I})), \quad w R_{2}=0.0988$ (all data). 11 [ $\mathrm{C}_{23} \mathrm{H}_{42} \mathrm{~N}_{2} \mathrm{OTi}$ ], $M=410.48$, orange crystal, $(0.280 \times 0.200 \times 0.100 \mathrm{~mm})$ orthorhombic, space group Pbca; $a=8.1915(3) \AA, b=23.6657(8) \AA, c$ $=24.0318(8) \AA ; \alpha=90^{\circ}, \beta=90^{\circ}, \gamma=90^{\circ}, V=4658.7(3) \AA^{3} ; Z=8 ; \mu=$ $0.382 \mathrm{~mm}^{-1} ; \rho_{\text {calc }}=1.170 \mathrm{~g} \cdot \mathrm{cm}^{-3} ; 82506$ reflexes $\left(\theta_{\max }=33.228^{\circ}\right), 8840$ unique $\left(R_{\text {int }}=0.0533\right) ; 255$ parameters; largest max. $/ \mathrm{min}$ in the final difference Fourier synthesis 0.757 e. $\AA^{-3 /} \quad-0.632$ e. $\AA^{-3}$; max./min. transmission $0.63 / 0.75 ; R_{1}=0.0458(\mathrm{I}>2 \sigma(\mathrm{I})), w R_{2}=0.1252$ (all data) 13: $\left[\mathrm{C}_{13.53} \mathrm{H}_{24.04} \mathrm{AICIN}{ }_{2} \mathrm{O}\right], M=293.24$, colorless crystal, $(0.281 \times 0.220 \times$ $0.172 \mathrm{~mm}$ ); monoclinic, space group $P 21 / c ; a=8.9805(9) \AA, b=$ 14.1884(11) $\AA, c=11.9568(10) \AA ; \alpha=90^{\circ}, \beta=90.216(4)^{\circ}, \gamma=90^{\circ}, V=$ $1523.5(2) \AA^{3} ; Z=4 ; \mu=0.302 \mathrm{~mm}^{-1} ; \rho_{\text {calc }}=1.278 \mathrm{~g} \cdot \mathrm{cm}^{-3} ; 37860$ reflections $\left(\theta_{\max }=33.207^{\circ}\right), 5631$ unique $\left(R_{\text {int }}=0.0263\right) ; 214$ parameters; largest max./min in the final difference Fourier synthesis $0.624 \mathrm{e} \cdot \AA^{-3} /-0.404 \mathrm{e} \cdot \AA^{-3} ;$ max./min. transmission $0.75 / 0.70 ; R_{1}=0.0440$ $(\mathrm{I}>2 \sigma(\mathrm{I})), w R_{2}=0.1220$ (all data). 14: $\left[\mathrm{C}_{9} \mathrm{H}_{17} \mathrm{AlCl}_{2} \mathrm{~N}_{2} \mathrm{O}\right], M=267.12$, colorless crystal, $(0.522 \times 0.202 \times 0.114 \mathrm{~mm})$; monoclinic, space group $P 21 / n ; a=6.9716(4) \AA, b=13.7557(7) \AA, c=13.3773(7) \AA ; \alpha=90^{\circ}, \beta=$ $100.188(2)^{\circ}, \gamma=90^{\circ}, V=1262.65(12) \AA^{3} ; Z=4 ; \mu=0.561 \mathrm{~mm}^{-1} ; \rho_{\text {calc }}=$ $1.405 \mathrm{~g} \cdot \mathrm{cm}^{-3} ; 72519$ reflexes $\left(\theta_{\max }=36.336^{\circ}\right), 5749$ unique $\left(R_{\text {int }}=\right.$ $0.0299) ; 140$ parameters; largest max./min in the final difference Fourier synthesis 0.617 e. $\AA^{-3} /-0.270$ e. $\AA^{-3}$; max./min. transmission $0.62 / 0.75 ; \quad R_{1}=0.0244(\mathrm{I}>2 \sigma(\mathrm{I})), \quad w R_{2}=0.0666$ (all data). 15 $\left[\mathrm{C}_{18} \mathrm{H}_{29} \mathrm{AlN}_{2} \mathrm{O}_{2}\right.$ ], $M=332.41$, colorless crystal, $(0.231 \times 0.161 \times 0.122$ $\mathrm{mm}$ ); orthorhombic, space group Pbca; $a=14.5393(6) \AA, \quad b=$ 15.3238(6) $\AA, c=16.6689(7) \AA ; \alpha=90^{\circ}, \beta=90^{\circ}, \gamma=90^{\circ}, V=3713.8(3)$ $\AA^{3} ; Z=8 ; \mu=0.120 \mathrm{~mm}^{-1} ; \rho_{\text {calc }}=1.189 \mathrm{~g} \cdot \mathrm{cm}^{-3} ; 59816$ reflexes $\left(\theta_{\max }=\right.$ $\left.33.153^{\circ}\right), 7091$ unique $\left(R_{\text {int }}=0.0377\right) ; 215$ parameters; largest $\max . / \mathrm{min}$ in the final difference Fourier synthesis $0.542 \mathrm{e} \cdot \AA^{-3} /-0.284$ e. $\AA^{-3}$; max./min. transmission $0.66 / 0.75 ; R_{1}=0.0388(\mathrm{I}>2 \sigma(\mathrm{I})), w R_{2}=$ 0.1135 (all data).

[12] a) C. K. Williams, L. E. Breyfogle, S. K. Choi, W. Nam, V. G. Young, Jr., M. A. Hillmyer, W. B. Tolman; J. Am. Chem. Soc. 2003, 125, 1135011359 ; b) C. Zhang, Z.-X. Wang, J. Organomet. Chem. 2008, 693, 3151-3158; c) L. Wang, H. Ma, Dalton Trans. 2010, 39, 7897-7910; d) C.-Y. Sung, C.-Y. Li, J.-K. Su, T.-Y. Chen, C.-H. Lin, B.-T. Ko, Dalton Trans. 2012, 41, 953-961; e) Y. Sun, Y. Cui, J. Xiong, Z. Dai, N. Tang J. Wu, Dalton Trans. 2015, 44, 16383-16391; f) M. Huang , C. Pan, H. Ma, Dalton Trans. 2015, 44, 12420-12431; g) T. Ebrahimi, E Mamleeva, I. Yu, S. G. Hatzikiriakos, P. Mehrkhodavandi, Inorg. Chem 2016, 55, 9445-9453; h) C. Kan, J. Hu, Y. Huang, H. Wang, H. Ma, Macromolecules 2017, 50, 7911-7919; i) Y. Huang, X. Kou, Y.-L. Duan, F.-F. Ding, Y.-F. Yin, W. Wang, Y. Yang, Dalton Trans. 2018, 47, 81218133.

[13] a) S. Schulz, R. Schäper, D. Bläser, C. Wölper, Z. Anorg. Allg. Chem. 2012, 638, 2102-2105; b) D. Bekermann, D. Rogalla, H.-W. Becker, M. Winter, R. A. Fischer, A. Devi, Eur. J. Inorg Chem. 2010, 1366-1372.

[14] X. Pang, X. Chen, X. Zhuang, X. Jing, J. Polym. Sci., Part A: Polym. Chem. 2008, 46, 643-649.

[15] H.-C. Tseng, M. Y. Chiang, W.-Y. Lu, Y.-J. Chen, C.-J. Lian, Y.-H. Chen, H.-Y. Tsai, Y.-C. Lai, H.-Y. Chen, Dalton Trans. 2015, 44, 1176311773.

[16] A. F. Lugo, A. F. Richards, Eur. J. Inorg. Chem. 2010, 2025-2035

[17] C. M. Beavers, G. H. Talbo, A. F. Richards, J. Organomet. Chem. 2011, 696, 2507-2511.

[18] a) S. Milione, G. Milano, L. Cavallo, Organometallics 2012, 31, 84988504 ; b) O. Eisenstein, H. Gérard, Structure, Bonding, and Reactivity of Organoaluminum Molecular Species: A Computational Perspective, in PATAl's Chemistry of Functional Groups, 2017,1-32, J. Wiley \& Sons.

[19] J. Lewiński, A. E. H. Wheatley, Simple Trivalent Organoaluminum Species: Perspectives on Structure, Bonding, and Reactivity, In Top. Organomet. Chem., Ed. S. Woodward, S. Dagorne, 2013, 41, 1-58.

[20] J. Lewiński, J. Zachara, I. Justyniak, Organometallics. 1997, 16, 4597 4605

[21] A CSD database search gave 73 hits including side-arm donorfunctionalized derivatives. Cambridge Structural Database, Version 5.39 (Nov. 2017), see also: F. H. Allen, Acta Cryst. 2002, B58, 380-388.

[22] J. Kim, J.-W. Hwang, Y. Kim, M. H. Lee, Y. Han, Y. Do, J. Organomet Chem. 2001, 620, 1-7.

[23] H. Bürger, W. Sawodny, U. Wannagat, J. Organomet. Chem. 1965, 3 , 113-120.

[24] M. Mena, P. Royo, R. Serrano, M. A. Pellinghelli, A. Tiripicchio, Organometallics 1989, 8, 476-482.

[25] G. R. Fulmer, A. J. M. Miller, N. H. Sherden, H. E. Gottlieb, A. Nudelman, B. M. Stoltz, J. E. Bercaw, K. I. Goldberg, Organometallics 2010, 29, 2176-2179.

[26] G. M. Sheldrick, Acta Crystallogr. 1990, A46, 467-473. 
[27] a) G. M. Sheldrick, SHELXL-2014, Program for the Refinement of Crystal Structures University of Göttingen, Göttingen (Germany) 2014 c) G. M. Sheldrick, Acta Crystallogr. 2008, A64, 112-122); d) shelXle, $A$
Qt GUI for SHELXL, C. B. Hübschle, G. M. Sheldrick, B. Dittrich, J. Appl. Cryst. 2011, 44, 1281-1284. 
Entry for the Table of Contents (Please choose one layout)

Layout 1:

\section{FULL PAPER}

Several heteroleptic metal complexes of group $4(\mathrm{Ti}, \mathrm{Zr}), 12(\mathrm{Zn})$ and $13(\mathrm{Al})$ containing side-arm donorfunctionalized tridentate $\beta$-ketoiminate ligands were synthesized and structurally characterized.

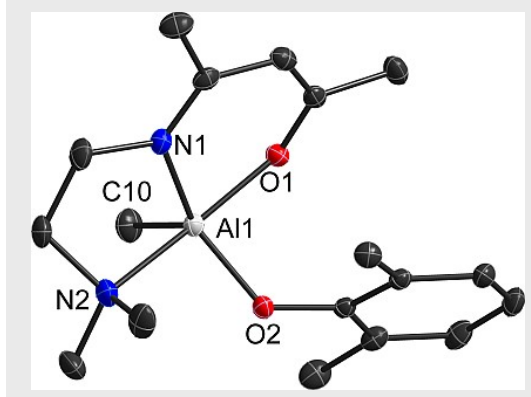

Dennis Dittrich, Phillip Steiniger, Christoph Wölper, and Stephan Schulz*

Page No. - Page No.

Synthesis and $X$-ray crystal structures of $\beta$-ketoiminate complexes 
Additional Author information for the electronic version of the article.

Prof. Dr. Stephan Schulz: ORCID identifier: 0000-0003-2896-4488 


\section{DuEPublico}

Duisburg-Essen Publications online
DNIVERSITAT

offen im Denken Ub $\begin{aligned} & \text { universitäts } \\ & \text { bibliothek }\end{aligned}$

This text is made available via DuEPublico, the institutional repository of the University of Duisburg-Essen. This version may eventually differ from another version distributed by a commercial publisher.

DOI: $\quad 10.1002 /$ zaac.201800321

URN: urn:nbn:de:hbz:464-20201218-104344-1

This is the peer reviewed version of the following article: Z. Anorg. Allg . Chem. 2018, $644,1367-1375$, which has been published in final form at:

https://doi.org/10.1002/zaac.200801382

All rights reserved. 